\title{
Learning Security Classifiers with Verified Global Robustness Properties
}

\author{
Yizheng Chen \\ UC Berkeley \\ 1z@berkeley.edu \\ Xiaojing Liao \\ Indiana University Bloomington \\ xliao@iu.edu
}

\author{
Shiqi Wang \\ Columbia University \\ tcwangshiqi@cs.columbia.edu \\ Suman Jana \\ Columbia University \\ suman@cs.columbia.edu
}

\author{
Yue Qin \\ Indiana University Bloomington \\ qinyue@iu.edu \\ David Wagner \\ UC Berkeley \\ daw@cs.berkeley.edu
}

\begin{abstract}
Many recent works have proposed methods to train classifiers with local robustness properties, which can provably eliminate classes of evasion attacks for most inputs, but not all inputs. Since data distribution shift is very common in security applications, e.g., often observed for malware detection, local robustness cannot guarantee that the property holds for unseen inputs at the time of deploying the classifier. Therefore, it is more desirable to enforce global robustness properties that hold for all inputs, which is strictly stronger than local robustness.

In this paper, we present a framework and tools for training classifiers that satisfy global robustness properties. We define new notions of global robustness that are more suitable for security classifiers. We design a novel booster-fixer training framework to enforce global robustness properties. We structure our classifier as an ensemble of logic rules and design a new verifier to verify the properties. In our training algorithm, the booster increases the classifier's capacity, and thefi xer enforces verified global robustness properties following counterexample guided inductive synthesis.

We show that we can train classifiers to satisfy different global robustness properties for three security datasets, and even multiple properties at the same time, with modest impact on the classifier's performance. For example, we train a Twitter spam account classifier to satisfyfi ve global robustness properties, with $5.4 \%$ decrease in true positive rate, and $0.1 \%$ increase in false positive rate, compared to a baseline XGBoost model that doesn't satisfy any property.
\end{abstract}

\section{CCS CONCEPTS}

- Security and privacy $\rightarrow$ Logic and verification; Malware and its mitigation; Social network security and privacy; Network security; • Computing methodologies $\rightarrow$ Machine learning algorithms; Logical and relational learning; Rule learning;

\footnotetext{
Permission to make digital or hard copies of all or part of this work for personal or classroom use is granted without fee provided that copies are not made or distributed for profit or commercial advantage and that copies bear this notice and the full citation on thefi rst page. Copyrights for components of this work owned by others than the author(s) must be honored. Abstracting with credit is permitted. To copy otherwise, or republish, to post on servers or to redistribute to lists, requires prior specific permission and/or a fee. Request permissions from permissions@acm.org. CCS '21, November 15-19, 2021, Virtual Event, Republic of Korea

(C) 2021 Copyright held by the owner/author(s). Publication rights licensed to ACM. ACM ISBN 978-1-4503-8454-4/21/11 ..\$15.00

https://doi.org/10.1145/3460120.3484776
}

\section{KEYWORDS}

Verifiable Machine Learning; Security Classifier; Adversarial machine learning; Global Robustness Properties; Formal Verification

ACM Reference Format:

Yizheng Chen, Shiqi Wang, Yue Qin, Xiaojing Liao, Suman Jana, and David Wagner. 2021. Learning Security Classifiers with Verified Global Robustness Properties. In Proceedings of the 2021 ACM SIGSAC Conference on Computer and Communications Security (CCS '21), November 15-19, 2021, Virtual Event, Republic of Korea. ACM, New York, NY, USA, 18 pages. https: //doi.org/10.1145/3460120.3484776

\section{INTRODUCTION}

Machine learning classifiers can achieve high accuracy to detect malware, spam, phishing, online fraud, etc., but they are brittle against evasion attacks. For example, to detect whether a Twitter account is spamming malicious URLs, many research works have proposed to use content-based features, such as the number of tweets containing URLs from that account [46, 48, 58, 82]. These features are useful at achieving high accuracy, but attackers can easily modify their behavior to evade the classifier.

In this paper, we develop a framework and tools for addressing this problem. First, the defenders identify a property $\varphi$ that the classifier should satisfy; typically, $\varphi$ identifies a class of evasion strategies that might be available to an adversary, and specifies a requirement on their effect on the classifier (e.g., that they won't change the classifier's output too much). Next, the defenders train a classifier $\mathcal{F}$ that satisfies $\varphi$. We identify several properties $\varphi$ that capture different notions of classifier robustness. Then, we design an algorithm for the classifier design problem:

Given a property $\varphi$ and a training set $\mathcal{D}$, train a classifier $\mathcal{F}$ that satisfies $\varphi$.

Our algorithm trains a verifiably robust classifier: we can formally verify that $\mathcal{F}$ satisfies $\varphi$.

Existing works focus on training and verifying local robustness properties of classifiers. Typically, they verify the following local robustness property: define $\varphi(x)$ to be the assertion that for all $x^{\prime}$, if $\left\|x^{\prime}-x\right\|_{p} \leq \epsilon$, then $\mathcal{F}\left(x^{\prime}\right)$ is classified the same as $\mathcal{F}(x)$. The past works provide a way to verify whether $\varphi(x)$ holds, for afi xed $x$, and then devise ways to train a classifier $\mathcal{F}$ so that $\varphi(x)$ holds for most $x$, but not all $x$ (local robustness). So far, the most promising defenses against adversarial examples all take this form, including adversarial training [59], certifiable training [19, 62, 85, 92], and randomized smoothing techniques $[14,45,51]$. To evaluate the local 
robustness of a trained model, we can measure the percentage of data points $x$ in the test set that satisfy $\varphi(x)[18,20,21,26,28,34$, $38,57,70,71,77,78,83,86,87,90,92]$. This measurement is useful if at the time of deploying the classifier, the real-world data follow the same distribution as the measured test set. Unfortunately, data distribution shift is very common in security applications (e.g., malware detection $[3,61,66])$, so local robustness cannot guarantee that the robustness property holds for most inputs at deployment time. In fact, in some cases, the adversary may be able to adapt their behavior by choosing novel samples $x$ that don't follow the test distribution and such that $\varphi(x)$ doesn't hold.

In this paper, we address these challenges by showing how to train classifiers that satisfy verified global robustness properties. We define a global robustness property as a universally quantified statement over one or more inputs to the classifier, and its corresponding outputs: e.g., $\forall x . \varphi(x)$ or $\forall x, x^{\prime} . \varphi\left(x, x^{\prime}\right)$. Since global robustness holds for all inputs, it is strictly stronger than local robustness, and it ensures robustness even under distribution shift. Then, we identify a number of robustness properties that may be useful in security applications, and we develop a general technique to achieve the properties. Our technique can handle a large class of properties, formally defined in Section 5.2.1. The vast majority of past work has focused on $\ell_{p}$ robustness, perhaps motivated by computer vision; however, in security settings, such as detecting malware, online fraud, or other attacks, other notions of robustness may be more appropriate.

There are many challenges in training classifiers with global robustness properties. First, it is hard to maintain good test accuracy since the definition of global robustness is much stronger than local robustness. To the best of our knowledge, among global robustness properties, only two properties have been previously achieved. One of them is monotonicity [35, 89]; and the other is a concurrent work that has proposed $\ell_{p}$-norm robustness with the option to abstain on non-robust inputs [50]. For example, researchers have trained a monotonic malware classifier to defend against evasion attacks that add content to a malware [35]. Monotonicity is useful: it limits the attacker to more expensive evasion operations that may remove malicious functionality from the malware, if they want to evade the classifier. However, monotonicity is not general enough to capture some types of evasion. Second, it is challenging to train classifiers with guarantees of global robustness. Several training techniques sacrifice global robustness in their algorithms. For example, DL2 [25] proposed several global robustness properties, but their training technique only achieves local robustness and cannot learn classifiers with global properties, because they rely on adversarial training. ART [56] presents an abstraction refinement method to train neural networks with global robustness properties. In principle ART can guarantee global robustness if the correctness loss reaches zero, however in their experiments the loss never reached zero.

To overcome these challenges, we design a novel booster-fixer training framework that enforces global robustness. Our classifier is structured as an ensemble of logic rules-a new architecture that is more expressive than trees given the same number of atoms and clauses (formally defined in Section 4.1)-and we show how to verify global robustness properties and then how to train them, for these ensembles. Intuitively, our algorithm trains a candidate classifier with good accuracy (but not necessarily any robustness), and then wefi $x$ the classifier to satisfy global robustness by iterativelyfinding counterexamples and repairing them using the Counterexample Guided Inductive Synthesis (CEGIS) paradigm [80]. Past works for training monotonic classifiers all use specialized techniques that do not generalize to other properties $[5,7,16,17,23,32,35,40,89]$. In contrast, our technique is fully general and can handle a large class of global robustness properties (formally defined in Section 5.2.1); we even show that we can enforce multiple properties at the same time (Section 3.2, Section 6.3.3).

We evaluate our approach on three security datasets: cryptojacking [41], Twitter spam accounts [47], and Twitter spam URLs [43]. Using security domain knowledge and results from measurement studies, we specify desirable global robustness properties for each classification task. We show that we can train all properties individually, and we can even enforce multiple properties at the same time, with a modest impact on the classifier's performance. For example, we train a classifier to detect Twitter spam accounts while satisfying five global robustness properties; the true positive rate decreases by $5.4 \%$ and the false positive rate increases by $0.1 \%$, compared to a baseline XGBoost model that doesn't satisfy any robustness property.

Since no existing work can train classifiers with any global robustness property other than monotonicity, we compare our approach against two types of baseline models: 1) monotonic classifiers, and 2) models trained with local versions of our proposed properties. For the monotonicity property, our results show that our method can achieve comparable or better model performance than prior methods that were specialized for monotonicity. We also verify that we can enforce each global robustness property we consider, which no prior method achieves for any of the other properties.

Our contributions are summarized as follows.

- We define new global robustness properties that are relevant for security applications.

- We design and implement a general booster-fixer training procedure to train classifiers with verified global robustness properties.

- We propose a new type of model, logic ensemble, that is well-suited to booster-fixer training. We show how to verify properties of such a model.

- We are thefi rst to train multiple global robustness properties. We demonstrate that we can enforce these properties while maintaining high test accuracy for detecting cryptojacking websites, Twitter spam accounts, and Twitter spam URLs. Our code is available at https://github.com/surrealyz/ verified-global-properties.

\section{EXAMPLE}

In this section, we present an illustrative example to show how our training algorithm works. Within our booster-fixer framework, thefi xer follows the Counterexample Guided Inductive Synthesis (CEGIS) paradigm. The key step in each CEGIS iteration is to start from a classifier without the global robustness property, use a verifier tofi nd counterexamples that violate the property, and train the classifier for one epoch guided by the counterexample. This process is repeated until the classifier satisfies the property. Here, we show how to train one CEGIS iteration for a classifier 


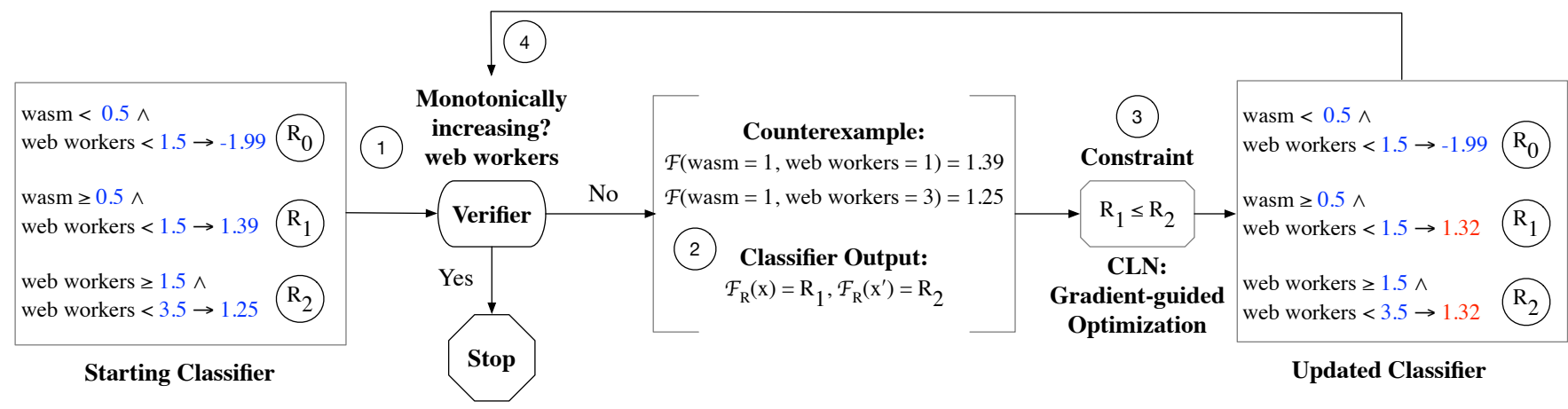

Figure 1: One CEGIS iteration of our training algorithm, illustrated on a simple example. Here we train a classifier to detect cryptojacking, while enforcing a monotonicity property.

to detect cryptojacking web pages. For simplicity, we demonstrate classification using only two features.

\subsection{Monotonicity for Cryptojacking Classifier}

We use two features to detect cryptojacking: whether the website uses WebAssembly (wasm), and the number of web workers used by the website [41]. Cryptojacking websites need the high performance provided by WebAssembly and often use multiple web worker threads to mine cryptocurrency concurrently. We enforce a monotonicity property for the web workers feature: the more web workers a website uses, the more suspicious it should be rated by the classifier, with all else held equal.

Figure 1 shows a single CEGIS iteration that starts from a classifier that violates the property, uses a counterexample to guide the training, and arrives at an updated classifier that satisfies the property. The classifier is structured as an ensemble of logic rules. For example, "wasm $<0.5 \wedge$ web workers $<1.5 \rightarrow-1.99$ " means that if the website does not use WebAssembly, and has at most one web worker, the clause adds $R \_0$ to thefi nal prediction value, which is currently -1.99 . Otherwise, the clause is inactive and adds nothing to thefi nal prediction value. The colored variables are learnable parameters. The classifier computes thefi nal score as a sum over all active clauses; if this score is greater than or equal to 0 , the webpage is classified as malicious.

Our training procedure executes the following steps:

Step (1), Figure 1: We use formal methods to verify whether the current classifier satisfies the monotonicity property for the web workers feature. If the property is verified, we have learned a robust classifier and the iteration stops. Otherwise, the verifier produces a counterexample that violates the property.

Step (2): We use clause return variables to represent the counterexample. The counterexample found by the verifier is $x=$ (wasm $=$ 1 , web workers $=1), x^{\prime}=($ wasm $=1$, web workers $=3)$, such that $x<x^{\prime}$ and $\mathcal{F}(x)>\mathcal{F}\left(x^{\prime}\right)$. We compute variables $\mathcal{F}_{R}(x)=R_{1}$, $\mathcal{F}_{R}\left(x^{\prime}\right)=R_{2}$ as the classifier output for each input, using the sum of return variables from the true clauses.

Step (3): We construct a logical constraint to represent that the counterexample from this pair of samples $\left(x, x^{\prime}\right)$ should no longer violate the monotonicity property, i.e., that $\mathcal{F}_{R}(x) \leq \mathcal{F}_{R}\left(x^{\prime}\right)$. Here, this is equivalent to $R_{1} \leq R_{2}$. Then, we re-train the classifier subject to the constraint that $R_{1} \leq R_{2}$. To enforce this constraint, we smooth the discrete classifier using Continuous Logic Networks (CLN) [72, 96], and then use projected gradient descent with the constraint to train the classifier. Gradient-guided optimization ensures that this counterexample $\left(x, x^{\prime}\right)$ will no longer violate the property and tries to achieve the highest accuracy subject to that constraint. After one epoch of training, the red parameters are changed by gradient descent in the updated classifier.

Lastly, we discretize the updated classifier and repeat the process again. In the second iteration, we query the verifier again (Step (4)). In this example, the updated classifier from thefi rst iteration satisfies the monotonicity property, and the process stops.

This simplified example illustrates the key ideas behind our training algorithm. Appendix A shows another example, illustrating that this process is general and can enforce a large class of properties on the classifier. We define the properties we can support in Section 5.2.1.

\section{MODEL SYNTHESIS PROBLEM}

In this section, we formulate the model synthesis problem mathematically, and then propose new global robustness properties based on security domain knowledge.

\subsection{Problem Formulation}

Our goal is to train a machine learning classifier $\mathcal{F}$ that satisfies a set of global robustness properties. Without loss of generality, we focus on binary classification in the problem definition; this can be extended to the multi-class scenario. The classifier $\mathcal{F}_{\theta}: \mathbb{R}^{n} \rightarrow \mathbb{R}$ maps a feature vector $x=\left[x_{1}, x_{2}, \ldots, x_{n}\right]$ with $n$ features to a real number. Here $\theta$ represent the trainable parameters of the classifier; we omit them from the notation when they are not relevant. The classifier predicts $\hat{y}=1$ if $\mathcal{F}(x) \geq 0$, otherwise $\hat{y}=0$. We use $\mathcal{F}(x)$ to represent the classification score, and $g(\mathcal{F}(x))$ to denote the normalized prediction probability for the positive class, where $g: \mathbb{R} \rightarrow[0,1]$. For example, we can use sigmoid as the normalized prediction function $g$. We formally define the model synthesis problem here.

Definition 3.1 (Model Synthesis Problem). A model synthesis problem is a tuple $(\Phi, \mathcal{D})$, where

- $\Phi$ is a set of global robustness properties, $\Phi=\left\{\varphi_{1}, \varphi_{2}, \ldots, \varphi_{k}\right\}$. 
- $\mathcal{D}$ is the training dataset containing $m$ training samples with their labels $\left(x^{(1)}, y^{(1)}\right), \ldots,\left(x^{(m)}, y^{(m)}\right)$.

Definition 3.2 (Solution to Model Synthesis Problem). A solution to the model synthesis problem $(\Phi, \mathcal{D})$ is a classifier $\mathcal{F}_{\theta}$ with weights $\theta$ that minimizes a loss function $\mathcal{L}$ over the training set, subject to the requirement that the classifier satisfies the global robustness properties $\Phi$.

$$
\begin{array}{r}
\theta=\underset{\theta}{\arg \min } \sum_{\mathcal{D}} \mathcal{L}\left(y, g\left(\mathcal{F}_{\theta}(x)\right)\right) \\
\text { subject to } \forall \varphi_{i} \in \Phi, \mathcal{F}_{\theta}=\varphi_{i}
\end{array}
$$

In Section 5, we present a novel training algorithm to solve the model synthesis problem.

\subsection{Global Robustness Property Definition}

We are interested in global robustness properties that are relevant for security classifiers. Below, we definefi ve general properties that allow us to incorporate domain knowledge about what is considered to be more suspicious, about what kinds of low-cost evasion strategies the attackers can use without expending too many resources, and about the semantics and dependency among features.

Property 1 (Monotonicity): Given a feature $j$,

$$
\forall x, x^{\prime} \in \mathbb{R}^{n} \cdot\left[x_{j} \leq x_{j}^{\prime} \wedge\left(\forall i \neq j . x_{i}=x_{i}^{\prime}\right)\right] \Longrightarrow \mathcal{F}(x) \leq \mathcal{F}\left(x^{\prime}\right)
$$

This property specifies that the classifier is monotonically increasing along some feature dimension. It is useful to defend against a class of attacks that insert benign features into malicious instances (e.g., mimicry attacks [84], PDF content injection attacks [44], gradientguided insertion-only attacks [31], Android app organ harvesting attacks [67]). If we carefully choose features to be monotonic for a classifier, injecting content into a malicious instance can only make it look more malicious to the classifier (not less), i.e., these changes can only increase (not decrease) classification score. Therefore, evading the classifier will require the attacker to adopt more sophisticated strategies, which may incur a higher cost to the attacker; also, in some settings, these strategies can potentially disrupt the malicious functionality of the instance, rendering it harmless.

A straightforward variant is to require that the prediction score be monotonically decreasing (instead of increasing) for some features. For example, we might specify that, all else being equal, the more followers a Twitter account has, the less likely it is to be malicious. It is cheap for an attacker to obtain a fake account with fewer followers, but expensive to buy a fake account with many followers or to increase the number of followers on an existing account. Therefore, by specifying that the prediction score should be monotonically decreasing in the number of followers, we force the attacker to spend more money if they wish to evade the classifier by perturbing this feature.

Property 2 (Stability): Given a feature $j$ and a constant $c$,

$$
\forall x, x^{\prime} \in \mathbb{R}^{n} .\left[\forall i \neq j . x_{i}=x_{i}^{\prime}\right] \Longrightarrow\left|\mathcal{F}(x)-\mathcal{F}\left(x^{\prime}\right)\right| \leq c
$$

The stability property states that for all $x, x^{\prime}$, if they only differ in the $j$-th feature, the difference between their prediction scores is bounded by a constant $c$. The stability constant $c$ is effectively a Lipschitz constant for dimension $j$ (when all other features are heldfi xed), when $x, x^{\prime}$ are compared using the $L_{0}$ distance:

$$
\left|\mathcal{F}(x)-\mathcal{F}\left(x^{\prime}\right)\right| \leq c\left\|x_{j}-x_{j}^{\prime}\right\|_{0}
$$

We can generalize the stability property definition to a subset of features $J$ that can be arbitrarily perturbed by the attacker.

$$
\forall x, x^{\prime} \in \mathbb{R}^{n} \cdot\left[\forall i \notin J . x_{i}=x_{i}^{\prime}\right] \Longrightarrow\left|\mathcal{F}(x)-\mathcal{F}\left(x^{\prime}\right)\right| \leq c\left\|x-x^{\prime}\right\|_{0}
$$

Researchers have shown that constraining the local Lipschitz constant to be small when training neural networks can increase the robustness against adversarial examples [12, 33]. However, existing training methods rely on regularization techniques and thus achieve only local robustness; they cannot enforce a global Lipschitz constant. We are interested in the $\ell_{0}$ distance, because some low-cost features can be trivially perturbed by the attacker to evade security classifiers: the attacker can replace the value of those features with any other desired value. The stability property captures this by allowing the stable feature to be arbitrarily changed.

Low-cost Features. Some features can have their values arbitrarily replaced without too much difficulty. We dub these low-cost features, because it does not cost the attacker much to arbitrarily modify the value of these features. In particular a low-cost feature is one that is trivial to change, i.e. does not require nontrivial time, effort, and economic cost to perturb. All other features are called high-cost. Section 6.1 gives a concrete analysis of which features are low-cost for three security datasets.

Property 3 (High Confidence): Given a set of low-cost features $J$,

$$
\forall x, x^{\prime} \in \mathbb{R}^{n} .\left[\forall i \notin J . x_{i}=x_{i}^{\prime}\right] \wedge g(\mathcal{F}(x)) \geq \delta \Longrightarrow \mathcal{F}\left(x^{\prime}\right) \geq 0
$$

The high confidence property states that, for any sample $x$ that is classified as malicious with high confidence (e.g., $\delta=0.98$ ), perturbing any low-cost feature $j \in J$ does not change the classifier prediction from malicious to benign. Many low-cost features in security applications are useful to increase accuracy in the absence of evasion attacks, but they can be easily changed by the attacker. For example, to evade cryptojacking detection, an attacker could use an alias of the hash function name, to evade the hash function feature. This property allows such features to influence the classification if the sample is near the decision boundary, but for samples classified as malicious with high confidence, modifying just low-cost features should not be enough to evade the classifier. Thus, samples detected with high confidence by the classifier will be immune to such low-cost evasion attacks.

Property 3a (Maximum Score Decrease): Given a set of lowcost features $J$,

$$
\forall x, x^{\prime} \in \mathbb{R}^{n} \cdot\left[\forall i \notin J . x_{i}=x_{i}^{\prime}\right] \Longrightarrow \mathcal{F}(x)-\mathcal{F}\left(x^{\prime}\right) \leq g^{-1}(\delta)
$$

Property $3 \mathrm{a}$ is stronger than Property 3. If the maximum decrease of any classification score is bounded by $g^{-1}(\delta)$, then any high confidence classification score does not drop below zero. We provide the proof in Appendix B. In Section 5.2, we design the training constraint for Property 3 a in order to train for Property 3 (Table 2). 
Property 4 (Redundancy): Given $M$ groups of low-cost features $J_{1}, J_{2}, \ldots, J_{M}$

$$
\begin{aligned}
& \forall x, x^{\prime} \in \mathbb{R}^{n} \cdot\left[\forall i \notin \bigcup_{m=1}^{M} J_{m} \cdot x_{i}=x_{i}^{\prime}\right] \wedge g(\mathcal{F}(x)) \geq \delta \\
& \wedge \neg\left[\forall m=1, \ldots, M, \exists j_{m}\right.\left.\in J_{m}, x_{j_{m}} \neq x_{j_{m}}^{\prime}\right] \\
& \Longrightarrow \mathcal{F}\left(x^{\prime}\right) \geq 0
\end{aligned}
$$

If the attacker perturbs multiple low-cost features, we would like the high confidence predictions from the classifier to be robust if different groups of low-cost features are not perturbed at the same time. In the redundancy property, we identify $M$ groups of low-cost features, and require that the attacker has to perturb at least one feature from each group in order to evade a high confidence prediction. In other words, this makes each group of low-cost features redundant of every other group. If we know all the high-cost features with any one group of low-cost features, all high confidence predictions are robust.

Property 5 (Small Neighborhood): Given a constant $c$,

$$
\forall x, x^{\prime} \in \mathbb{R}^{n} \cdot d\left(x, x^{\prime}\right) \leq \epsilon \Longrightarrow\left|\mathcal{F}(x)-\mathcal{F}\left(x^{\prime}\right)\right| \leq c \cdot \epsilon
$$

where $d\left(x, x^{\prime}\right)=\max _{i}\left\{\left|x_{i}-x_{i}^{\prime}\right| / \sigma_{i}\right\}$.

The small neighborhood property specifies that for any two data points within a small neighborhood defined by $d$, we want the classifier's output to be stable. We define the neighborhood by a new distance metric $d\left(x, x^{\prime}\right)$ that measures the largest change to any feature value, normalized by the standard deviation of that input feature. $d\left(x, x^{\prime}\right)$ is essentially a $\ell_{\infty}$ norm, applied to normalized feature values. We chose not to use the $\ell_{\infty}$ distance directly because different features for security classifiers often have a different scale.

\section{PROPERTY VERIFICATION}

In this section, we describe the key ingredients we need to solve the model synthesis problem. We define a new type of classifier that is well-suited to model synthesis, and a verification algorithm to verify whether the classifier satisfies the properties.

\subsection{Logic Ensemble Classifier}

We propose a new type of classification model, which we call a logic ensemble. We show how to train logic ensemble classifiers that satisfy global robustness properties.

Definition 4.1 (Logic Ensemble Definition). A logic ensemble classifier $\mathcal{F}$ consists of a set of clauses. Each clause has the form

$$
B_{1}\left(\alpha_{1}, \beta_{1}\right) \wedge B_{2}\left(\alpha_{2}, \beta_{2}\right) \wedge \cdots \wedge B_{m}\left(\alpha_{m}, \beta_{m}\right) \rightarrow R
$$

where $B_{1} \ldots B_{m}$ are atoms and $R$ is the activation value of the clause. Each atom $B_{i}$ has the form $\alpha_{i} x_{j}<\beta_{i}$ for some $j$. Here the $\alpha_{i}, \beta_{i}, R$ are trainable parameters for the classifier. The implication denotes that if the body of the clause holds (all atoms $B_{1} \ldots B_{m}$ are true), then the clause returns an activation value $R$, otherwise it returns 0 . The classifier's output is computed as $\mathcal{F}_{\alpha, \beta, R}(x)=\sum R_{i}$, where the sum is over all clauses that are satisfied by $x$.

Logic ensembles can be viewed as a generalization of decision trees. Any decision tree (or ensemble of trees) can be expressed as a logic ensemble, with one clause per leaf in the tree, but logic ensembles are more expressive (for afi xed number of clauses) because they can also represent other structures of rules. Researchers have previously shown how to train decision trees with monotonicity properties, so our work can be viewed as an extension of this to a more expressive class of classifiers and a demonstration that this allows enforcing other robustness properties as well.

\subsection{Integer Linear Program Verifier}

We present a new verification algorithm that uses integer linear programming to verify the global robustness properties of logic ensembles, including trees. First, we encode the logic ensemble using boolean variables, adding consistency constraints among the boolean variables. Then, for each global robustness property, we symbolically represent the input and output of the classifier in terms of these boolean variables, and add extra constraints to assert that the robustness property is violated. Next, we check feasibility of these constraints, expressing them as a $0 / 1$ integer linear program. If an ILP solver canfi nd a feasible solution, the classifier does not satisfy the corresponding global robustness property, and the solver will give us a counterexample. On the other hand, if the integer linear program is infeasible, the classifier satisfies the global robustness property.

We describe our algorithm in more detail below. We use the binary variables in the $0 / 1$ integer linear program to represent an arbitrary input $x$ :

Atom ( $p)$ : We use $p_{i}$ variables to encode the truth value of atoms. Each atom is transformed into the same form of predicate $x_{j}<\eta_{i}$. Therefore, each predicate variable $p_{i}$ is associated with a feature dimension $j$ and the inequality threshold $\eta_{i}$.

Clause Status ( $(l)$ : We use $l_{k}$ variables to encode the truth value of clauses. When $l_{k}=1$, all atoms in the $k$-th clause are true, and the clause adds $R_{k}$ activation value for the classifier output.

Auxiliary Variables (a): We use $a_{i 1}$ and $a_{i 2}$ variables to encode the neighborhood range for the small neighborhood property, defined in Equation (8). For each predicate $x_{j}<\eta_{i}$, we create $a_{i_{1}}$ variable for $x_{j}<\eta_{i}-\sigma_{j} * \epsilon$, and $a_{i_{2}}$ variable for $x_{j}<\eta_{i}+\sigma_{j} * \epsilon$. If $x_{j}$ is within [ $\left.\eta_{i}-\sigma_{j} * \epsilon, \eta_{i}+\sigma_{j} * \epsilon\right]$, we must have $a_{i_{2}}-a_{i_{1}}=0$.

Double Variables: All the aforementioned variables are doubled as $p^{\prime}, l^{\prime}, a^{\prime}$ to represent the perturbed input $x^{\prime}$ bounded by the robustness property definition. The classifier's output for arbitrary $x, x^{\prime}$ are:

$$
\mathcal{F}(x)=\sum_{k} l_{k} R_{k}, \mathcal{F}\left(x^{\prime}\right)=\sum_{k} l_{k}^{\prime} R_{k}
$$

Then, we create the following linear constraints to ensure dependency between variables of the classifier.

Integer Constraints: We merge predicates for integer features. For example, if $x_{5}$ is an integer feature, we use the same binary variable to represent atoms $x_{5}<0.2$ and $x_{5}<0.3$.

Predicate Consistency Constraints: Predicate variables for the same feature dimension are sorted and constrained accordingly. For any $p_{i}, p_{t}$ belonging to the same feature dimension with $\eta_{i}<\eta_{t}$, $x_{j}<\eta_{t}$ must be true if $x_{j}<\eta_{i}$ is true. Thus, we have $p_{i} \leq p_{t}$.

Redundant Predicate Constraints: We set redundant variables to be always 0 . For example, $x_{j}<0$ is always false for a nonnegative feature.

Lastly, we use standard boolean encoding for Property Violation Constraints to verify a given property, as shown in Table 1. 


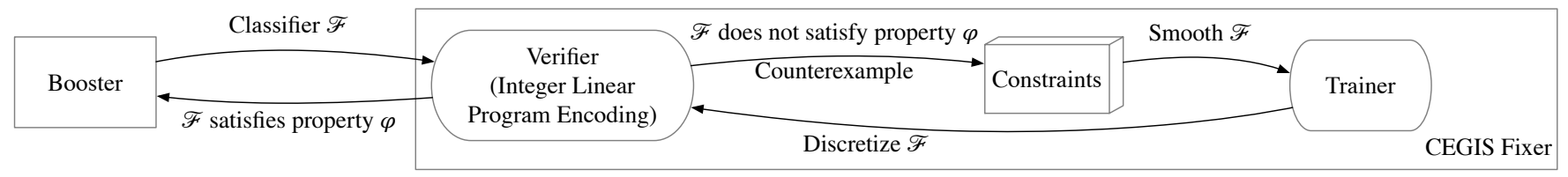

Figure 2: Booster-fixer training framework.

Each property has a pair of input and output constraints. In addition, we encode input consistency constraints for a given property.

Input Consistency Constraints: For any $x_{i}$ and $x_{i}^{\prime}$, if they are defined to be the same by the property, we set the related predicate variables $p$ and $p^{\prime}$ to have the same value.

Monotonicity: For two arbitrary inputs $x$ and $x^{\prime}$, if $x<x^{\prime}$, then there must be at least one more predicate true for $x^{\prime}$. The output constraint for (1) denotes violation to monotonically non-decreasing output, and (2) denotes violation to monotonically non-increasing output.

Stability: The input constraint says $x$ and $x^{\prime}$ are different, and the output constraint says the difference between $\mathcal{F}(x)$ and $\mathcal{F}\left(x^{\prime}\right)$ are larger than the stable constant $c_{\text {stability. }}$

High Confidence: The input constraint says $x$ is classified as malicious with at least $\delta$ confidence. The output constraints says $x^{\prime}$ is classified as benign.

Redundancy: The input and output constraints are the same as high confidence property. However, we encode predicate consistency constraints differently. We set variable equality constraints such that $x_{i}=x_{i}^{\prime}$ for $i$ outside the $M$ low-cost feature groups. We encode the disjunction of the conditions that only features from the same group are changed.

Small Neighborhood: For input constraint, for each $j$, wefirst encode the conjunction that $x_{j}$ and $x_{j}^{\prime}$ are both within a small neighborhood interval $a_{i_{2}}-a_{i_{1}}=1$. Then, we encode the disjunction that $x_{j}$ and $x_{j}^{\prime}$ can be only within one of such intervals surrounding the predicates. The output constraint says the difference between the outputs are larger than the allowed range.

\section{TRAINING ALGORITHM}

\subsection{Framework}

Figure 2 gives an overview of our booster-fixer training framework. We have two major components, a booster and afi xer, which interact with each other to train a classifier with high accuracy that satisfies the global robustness properties.

The booster increases the size of the classifier, and improves classification performance. We run $N$ boosting rounds. The classifier is a sum of logic ensembles, $\mathcal{F}(x)=\sum_{b=1}^{N} f_{b}(x)$, where each $f_{b}$ is a logic ensemble. In the $b$-th boosting round, the booster adds $f_{b}$ to the ensemble, proposing a candidate classifier $\sum_{i=1}^{b} f_{i}(x)$ (which does not need to satisfy any robustness property); then thefi xerfi xes property violations for this classifier. Empirically, more boosting rounds typically lead to better test accuracy afterfi xing the properties.

Thefi xer uses counterexample guided inductive synthesis (CEGIS) tofi $x$ the global robustness properties for the current classifier. We use a verifier and a trainer to iteratively train the classifier, eliminating counterexamples in each iteration, until the classifier satisfies

\begin{tabular}{l|c}
\hline Property & Property Violation Constraints \\
\hline \hline \multirow{4}{*}{ Monotonicity } & (1) In: $\sum_{x} p_{i} \leq \sum_{x^{\prime}} p_{i}^{\prime}+1$, \\
& Out: $\sum_{x} l_{k} * R_{k}>\sum_{x^{\prime}} l_{k}^{\prime} * R_{k}$ \\
\cline { 2 - 2 } Stability & (2) In: $\sum_{x} p_{i} \leq \sum_{x^{\prime}} p_{i}^{\prime}+1$, \\
& Out: $\sum_{x} l_{k} * R_{k}<\sum_{x^{\prime}} l_{k}^{\prime} * R_{k}$ \\
\hline \multirow{2}{*}{ High } & In: $\left|\sum_{x} p_{i}-\sum_{x^{\prime}} p_{i}^{\prime}\right| \geq 1$, \\
Confidence & Out: $\left|\sum_{x} l_{k} * R_{k}-\sum_{x^{\prime}} l_{k}^{\prime} * R_{k}\right|>c_{\text {stability }}$ \\
\hline Redundancy & In: $\sum_{x} l_{k} * R_{k} \geq g^{-1}(\delta)$, \\
Out: $\sum_{x^{\prime}} l_{k}^{\prime} * R_{k}<0$
\end{tabular}

Table 1: Property violation constraints for the verifier.

the properties. In each CEGIS iteration, wefi rst use the verifier tofi nd a counterexample that violates the property. Then, we use training constraints to eliminate the counterexample. The training constraints reduce the space of candidate classifiers and make progress towards satisfying the property. We accumulate the training constraints over the CEGIS iterations, so that our classifier is guaranteed to satisfy global robustness properties when thefixer returns a solution. After wefi $\mathrm{x}$ the global robustness properties for the classifier $\sum_{i=1}^{b} f_{i}(x)$, we go back to boost the next $b+1$ round, to further improve the test accuracy. We will discuss the details of our training algorithm next.

\subsection{Robust Training Algorithm}

Algorithm 1 presents the pseudo-code for our global robustness training algorithm. As inputs, the algorithm needs specifications of the global robustness properties $\Phi$ (Section 3.2) and a training dataset $\mathcal{D}$ to train for both robustness and accuracy. In addition, we need a booster $\mathcal{B}$ (Section 5.1), a verifier $\mathcal{V}$ (Section 4.2), a trainer $\mathcal{S}$ (described below) and a loss function $\mathcal{L}$ to run the booster-fixer rounds. We can specify the number of boosting rounds $N$. The algorithm outputs a classifier $\mathcal{F}$ that satisfies all the specified global robustness properties.

First, our algorithm initializes an empty ensemble classifier $\mathcal{F}$ such that we can add sub-classifiers into it over the boosting rounds (Line 1). We also initialize an empty set of constraints $C$ (Line 2). Then, we go through $N$ rounds of boosting in the for loop from 


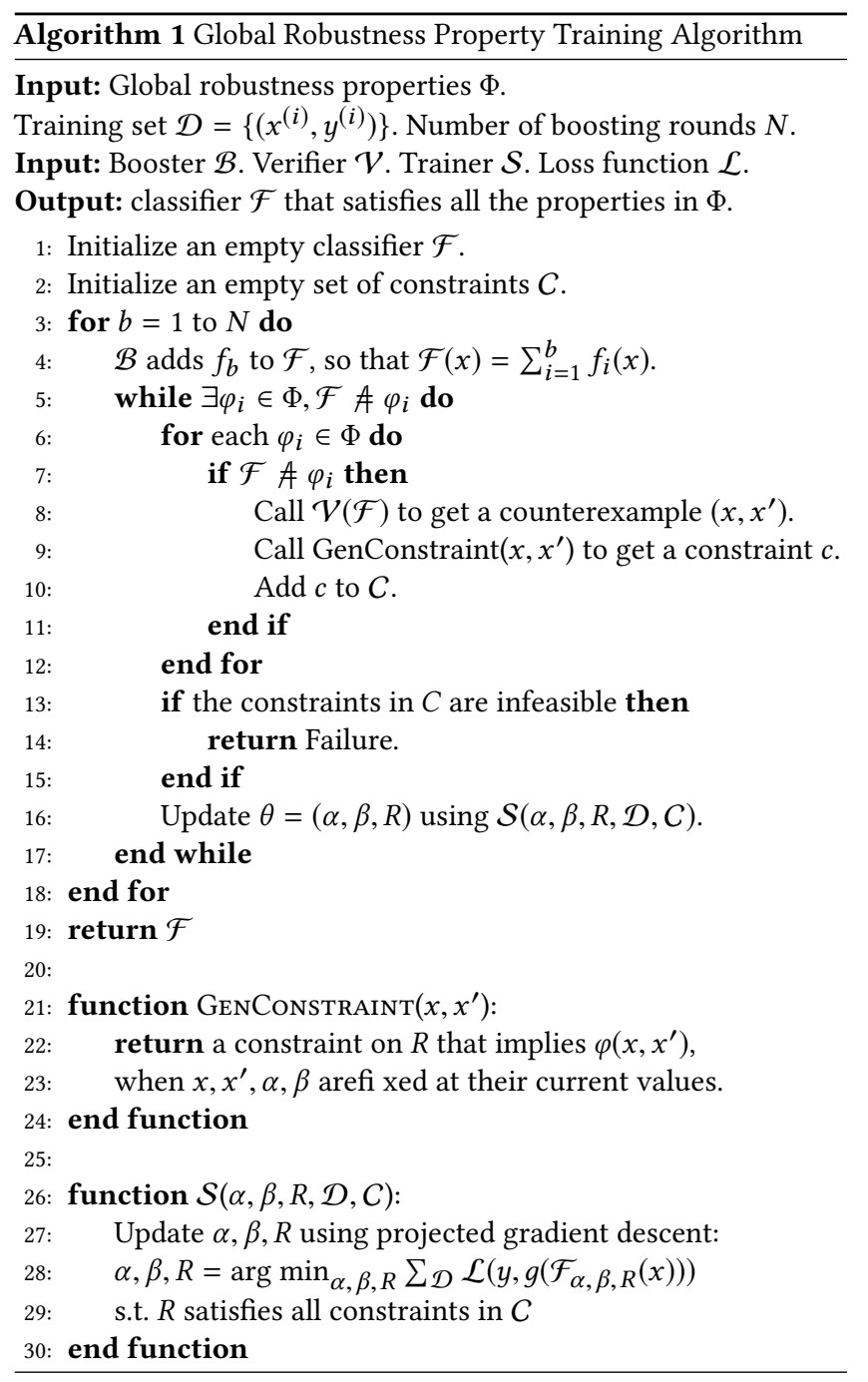

Line 3 to Line 18 . Within each boosting round $b$, the booster $\mathcal{B}$ adds a tree to the ensemble classifier, such that the current classifier is $\mathcal{F}(x)=\sum_{i=1}^{b} f_{i}(x)$. Thefi xer runs the while loop from Line 5 and Line 17. As long as the classifier does not satisfy all specified global robustness properties, we proceed withfi xing the properties (Line 5 ). For each property, if the model does not satisfy the property, the verifier $\mathcal{V}$ produces a counterexample $\left(x, x^{\prime}\right)$ (Line 8). Then, we generate a constraint $c$ that can eliminate the counterexample by calling a procedure GenConstraint $\left(x, x^{\prime}\right)$ (Line 9). We add the constraint to the set $C$. If the set of constraints are infeasible, the algorithm returns failure. Otherwise, we use the trainer $\mathcal{S}$ to train the weights $\theta$ using projected gradient descent (Line 16 calls $\mathcal{S}(\alpha, \beta, R, \mathcal{D}))$. We follow the gradient of the loss function w.r.t. the weights $\theta$, update the weights, and then we project the weights onto the $\ell_{2}$ norm ball centered around updated weights, subject to all constraints in $C$,. Therefore, the weights satisfy all constraints in $C$.

Generating Constraint. The GenConstraint function generates a constraint according to counterexample $\left(x, x^{\prime}\right)$. We use $\mathcal{F}_{R}(x)$ to represent the equivalence class of $x$ : all inputs that are classified the same as $x$, i.e., their classification score is a sum of return values

\begin{tabular}{l|c}
\hline Property & Training Constraints \\
\hline \hline \multirow{2}{*}{ Monotonicity } & $(1) \mathcal{F}_{R}(x) \leq \mathcal{F}_{R}\left(x^{\prime}\right)$ \\
\cline { 2 - 2 } & $(2) \mathcal{F}_{R}(x) \geq \mathcal{F}_{R}\left(x^{\prime}\right)$ \\
\hline Stability & $\left|\mathcal{F}_{R}(x)-\mathcal{F}_{R}\left(x^{\prime}\right)\right| \leq c_{\text {stability }}$ \\
\hline High Confidence & $\mathcal{F}_{R}(x)-\mathcal{F}_{R}\left(x^{\prime}\right)<g^{-1}(\delta)$ \\
\hline Redundancy & Same as high confidence. \\
\hline Small Neighborhood & $\left|\mathcal{F}_{R}(x)-\mathcal{F}_{R}\left(x^{\prime}\right)\right| \leq \epsilon * c_{\text {neighbor }}$ \\
\hline
\end{tabular}

Table 2: Constraints used for the training algorithm.

for the same set of clauses as $x$. We can use constraints over $\mathcal{F}_{R}(x)$ and $\mathcal{F}_{R}\left(x^{\prime}\right)$ to capture the change in the classifier's output, to satisfy the global robustness property for all counterexamples in the equivalence class. Specifically, in Table 2, we list the constraints forfi ve properties we have proposed. The constraints for monotonicity, stability, redundancy, and the small neighborhood properties have the same form as the output requirement specified in the corresponding property definitions. For the high confidence property, our training constraint is to bound the drop of the classification score to be no more than the $g^{-1}$ of the high confidence threshold $\delta$. This constraint aims to satisfy Property 3 a (Equation 6), which then satisfies Property 3 high confidence (Lemma 1 ). This constraint eliminates counterexamples faster than using the constraint $\mathcal{F}_{R}\left(x^{\prime}\right) \geq 0$.

CLN Trainer. Within thefi xer, we use Continuous Logic Networks (CLN) [72] to train the classifier to satisfy all constraints in $C$. If we directly enforce constraints over the weights of the classifier, the structure and weights will not have good accuracy. We want to use gradient-guided optimization to preserve accuracy of the classifier while satisfying the constraints. Since our discrete ensemble classifier is non-differentiable, wefi rst use CLN to smooth the logic ensemble. Following Ryan et al. [72], we use a shifted and scaled sigmoid function to smooth the inequalities, product $\mathrm{t}$-norm to smooth conjunctions. To train the smoothed model, we use binary cross-entropy loss as the loss function $\mathcal{L}$ for classification, and minimize the loss using projected gradient descent according to the constraints $C$. After training, we discretize the model back to logic ensemble for prediction, so we can verify the robustness properties. Note that although our training constraints $C$ are only related to the returned activation values of the clauses (Table 2), the learnable parameters of atoms may change as well due to the projection (See Appendix A for an example). In some cases, the structure of the atom can change as well. For example, if an atom $x_{0}<5$ is trained to become $-0.5 * x_{0}<2$, this changes the inequality of the atom.

5.2.1 Supported Properties. Our framework can handle any global robustness property $\varphi$ of the form $\forall x_{1}, \ldots, x_{k} \cdot \mu\left(x_{1}, \ldots, x_{k}\right) \Longrightarrow$ $v\left(\mathcal{F}\left(x_{1}\right), \ldots, \mathcal{F}\left(x_{k}\right)\right)$ where the set of values $\left\{\left(y_{1}, \ldots, y_{k}\right): v\left(y_{1}\right.\right.$, $\left.\left.\ldots, y_{k}\right)\right\}$ is a convex set, as then we can project the classifier weights accordingly (line 27 to line 29 in Algorithm 1). For example, for the monotonicity property, $k=2, \mu\left(x_{1}, x_{2}\right):=x_{1} \leq x_{2}$, and $v\left(\mathcal{F}\left(x_{1}\right), \mathcal{F}\left(x_{2}\right)\right):=\mathcal{F}\left(x_{1}\right) \leq \mathcal{F}\left(x_{2}\right)$. This class includes but is not limited to all global robustness properties with arbitrary linear constraints on the outputs of the classifier.

5.2.2 Algorithm Termination. Algorithm 1 is guaranteed to terminate. When the algorithm terminates, if itfi nds a classifier, the classifier is guaranteed to satisfy the properties. However, there is no guarantee that it willfi nd a classifier (line 14 of Algorithm 1 
returns Failure), but empirically our algorithm canfi nd an accurate classifier that satisfies all the specified properties, as shown in the results in Section 6.3.

\section{EVALUATION}

\subsection{Datasets and Property Specifications}

We evaluate how well our training technique works on three security datasets of different scale: detection of cryptojacking [41], Twitter spam accounts [47], and Twitter spam URLs [43]. Table 4 shows the size of the datasets. In total, the three datasets have $4 \mathrm{~K}$, $40 \mathrm{~K}$, and 422,672 data points respectively. Appendix D lists all the features for the three datasets. We specify global robustness properties for each dataset (Table 3) based on our analysis of what kinds of evasion strategies might be relatively easy and inexpensive for attackers to perform.

Monotonic Directions. To specify monotonicity properties, we use two types of security domain knowledge, suspiciousness and economic cost. We specify a classifier to be monotonically increasing for a feature if, (1) an input is more suspicious as the feature value increases, or, (2) a feature requires a lot of money to be decreased but easier to be increased, such that we force the attackers to spend more money in order to reduce the classification score. Similarly, we specify a classifier to be monotonically decreasing along a feature dimension by analyzing these two aspects.

6.1.1 Cryptojacking. Crytpojacking websites are malicious webpages that hijack user's computing power to mine cryptocurrencies. Kharraz et al. [41] collected cryptojacking website data from 12 families of mining libraries. We randomly split the dataset containing 2000 malicious websites and 2000 benign websites into $70 \%$ training set and $30 \%$ testing data. In total, there are 2800 training samples and 1200 testing samples. We use the training set as the validation set. Low-cost feature. Among all features, only the hash function feature is low cost to change. The attacker may use a hash function not on the list, or may construct aliases of the hash functions to evade the detection. Since the other features are related to usage of standard APIs or essential to running high performance cryptocurrency mining code, they are not trivial to evade.

Monotonicity. We specify all features to be monotonically increasing. Kharraz et al. [41] proposed seven features to classify cryptojacking websites. A website is more suspicious if any of these features have larger values. Specifically, cryptojacking websites prefer to use WebSocket APIs to reduce network communication bandwidth, use WebAssembly to run mining code faster, runs parallel mining tasks, and may use a list of hash functions. Also, if a website uses more web workers, has higher messageloop load, and PostMessage event load, it is more suspicious are performing some heavy load operations such as coin mining.

Stability. Since this is a small dataset, we specify all features to be stable, with stable constant 0.1 .

High Confidence. We use high confidence threshold 0.98.

Small Neighborhood. We specify $\epsilon=0.2, c=0.5$. Each feature is allowed to be perturbed by up to $20 \%$ of its standard deviation, and the output of the classifier is bounded by 0.01 .

6.1.2 Twitter Spam Accounts. Lee et al. [47] used social honeypot to collect information about Twitter spam accounts, and randomly sampled benign Twitter users. We reimplement 15 of their proposed features, including account age, number of following, number of followers, etc., with the entire list in Table 11, Appendix D. We randomly split the dataset into 36,000 training samples and 4,000 testing samples, and we use the training set as validation set.

Economic Cost Measurement Study. We have crawled and analyzed 6,125 for-sale Twitter account posts from an underground forum to measure the effect of LenScreenName and NumFollowers on the prices of the accounts.

- LenScreenName. Accounts with at most 4 characters are deemed special in the underground forum, usually on sale with a special tag ' $3-4$ Characters'. Table 5 shows that the average price of accounts with at most 4 characters isfi ve times the price of accounts with more characters or unspecified characters. More measurement results are in Appendix C.1.

- NumFollowers. We measure the account price distribution according to different tiers of followers indicated in the underground forum, from $500,1 \mathrm{~K}, 2 \mathrm{~K}$ up to $250 \mathrm{~K}$ followers. As shown in Figure 3, the account prices increase as the number of followers increases.

Low-cost Features. We identify 8 low-cost features in total. Among them, two features are related to the user profile, LenScreenName and LenProfileDescription. According to our economic cost measurement study, accounts with user names up to 4 characters are considered high cost to obtain. Therefore, we specify LenScreenName with at least 5 characters to be low cost feature range. The other four low-cost features are related to the tweet content, since they can be trivially modified by the attacker: NumTweets, NumDailyTweets, TweetLinkRatio, TweetUniqLinkRatio, TweetAtRatio, and TweetUniqAtRatio.

Monotonicity. We specify two features to be monotonically increasing, and two features to be monotonically decreasing, based on domain knowledge about suspicious behavior and economic cost measurement studies.

Increase in suspiciousness: Spammers tend to follow a lot of people, expecting social reciprocity to gain followers for spam content, so large NumFollowings makes an account more suspicious. If an account sends a lot of links (TweetLinkRatio and TweetUniqLinkRatio), it also becomes more suspicious.

Decrease in suspiciousness: Since cybercriminals are constantly trying to evade blocklists, if an account is newly registered with a small AgeDays value, it is more suspicious.

Increase in economic cost: Since the attacker needs to spend more money to obtain Twitter accounts with very few characters, we specify the LenScreenName to be monotonically increasing,

Decrease in economic cost: Since it is expensive for attackers to obtain more followers, we specify the NumFollowers feature to be monotonically decreasing.

Stability. We specify all the low-cost features to be stable, with stable constant 8 .

High Confidence. We allow the attacker to modify any one of the low cost features individually, but not together. We use a high confidence prediction threshold 0.98 .

Redundancy. Among the 8 low-cost features, we identify four groups, where each group has one feature that counts an item in total, and one other feature that counts the same item in a different 


\begin{tabular}{|c|c|c|}
\hline Dataset & Property & Specification \\
\hline \multirow{6}{*}{ Cryptojacking } & - & Low-cost features: whether a website uses one of the hash functions on the list. \\
\hline & Monotonicity & Increasing: all features \\
\hline & Stability & All features are stable. Stable constant $=0.1$ \\
\hline & High Confidence & $\delta=0.98$ \\
\hline & Small Neighborhood & $\epsilon=0.2, c=0.5$ \\
\hline & Combined & Monotonicity, stability, high confidence, and small neighborhood \\
\hline \multirow{7}{*}{$\begin{array}{l}\text { Twitter Spam } \\
\text { Accounts }\end{array}$} & - & $\begin{array}{l}\text { Low-cost features: LenScreenName ( } \geq 5 \text { char), LenProfileDescription, NumTweets, NumDailyTweets, } \\
\text { TweetLinkRatio, TweetUniqLinkRatio, TweetAtRatio, TweetUniqAtRatio. }\end{array}$ \\
\hline & Monotonicity & $\begin{array}{l}\text { Increasing: LenScreenName, NumFollowings, TweetLinkRatio, TweetUniqLinkRatio } \\
\text { Decreasing: AgeDays, NumFollowers }\end{array}$ \\
\hline & Stability & Low-cost features are stable. Stable constant $=8$ \\
\hline & High Confidence & $\delta=0.98$. Attacker is allowed to perturb any one of the low-cost features, but not multiple ones. \\
\hline & Redundancy & $\begin{array}{l}\delta=0.98, M=2 \text {, any } 2 \text { in } 4 \text { groups satisfy redundancy: } 1 \text { ) LenScreenName ( } \geq 5 \text { char), LenProfileDescription } \\
\text { 2) NumTweets, NumDailyTweets } 3 \text { ) TweetLinkRatio, TweetUniqLinkRatio } 4 \text { ) TweetAtRatio, TweetUniqAtRatio }\end{array}$ \\
\hline & Small Neighborhood & $\epsilon=0.1, c=50$ \\
\hline & Combined & Monotonicity, stability, high confidence, redundancy, and small neighborhood \\
\hline \multirow{5}{*}{$\begin{array}{l}\text { Twitter Spam } \\
\text { URLs }\end{array}$} & - & Low-cost features: Mention Count, Hashtag Count, Tweet Count, URL Percent. \\
\hline & Monotonicity & $\begin{array}{l}\text { Increasing: } 7 \text { shared resources features. EntryURLid, AvgURLid, ChainWeight, } \\
\text { CCsize, MinRCLen, AvgLdURLDom, AvgURLDom }\end{array}$ \\
\hline & Stability & Low-cost features are stable. Stable constant $=8$ \\
\hline & High Confidence & $\delta=0.98$. Attacker is allowed to perturb any one of the low-cost features, but not multiple ones. \\
\hline & Small Neighborhood & $\epsilon=1.5, c=10$ \\
\hline
\end{tabular}

Table 3: Global robustness property specifications for three datasets.

\begin{tabular}{c|r|r|r|r}
\hline Dataset & $\begin{array}{c}\text { Training } \\
\text { set size }\end{array}$ & $\begin{array}{c}\text { Test } \\
\text { set size }\end{array}$ & $\begin{array}{c}\text { Validation } \\
\text { set size }\end{array}$ & $\begin{array}{c}\text { \# of } \\
\text { features }\end{array}$ \\
\hline \hline Cryptojacking [41] & 2800 & 1200 & Train & 7 \\
\hline $\begin{array}{c}\text { Twitter Spam } \\
\text { Accounts [47] }\end{array}$ & 36,000 & 4,000 & Train & 15 \\
\hline $\begin{array}{c}\text { Twitter Spam } \\
\text { URLs [43] }\end{array}$ & 295,870 & 63,401 & 63,401 & 25 \\
\hline
\end{tabular}

Table 4: The three datasets we use to evaluate our methods. For cryptojacking and Twitter spam account datasets, we use the training set as the validation set.

granularity (daily or unique count). We specify that any two groups are redundancy of each other $(M=2)$ with $\delta=0.98$.

Small Neighborhood. We specify $\epsilon=0.1, c=50$. The attacker can change each feature up to $10 \%$ of its standard deviation value, and the classifier output change is bounded by 5 .

6.1.3 Twitter Spam URLs. Kwon et al. [43] crawled 15,828,532 tweets by $1,080,466$ users. They proposed to use URL redirection chains and and graph related features to classify spam URL posted on Twitter. We obtain their public dataset and re-extract 25 features according to the description in the paper. We extract four categories of features. (1) Shared resources features capture that the attacker reuse resources such as hosting servers and redirectors. (2) Heterogeneity-driven features reflect that attack resources may be heterogeneous and located around the world. (3) Flexibility-driven features capture that attackers use different domains and initial URLs to evade blocklists. (4) Tweet content features measure the number of special characters, tweets, percentage of URLs made by

\begin{tabular}{c|c}
\hline \# Char & Price \\
\hline \hline$\leq 4$ & $\$ 1,598.09$ \\
\hline$\geq 5$ & $\$ 298.40$ \\
\hline Unspecified & $\$ 147.62$
\end{tabular}

Table 5: Average price of for-sale Twitter accounts with different number of characters for the username.

the same user. This is the largest dataset in our evaluation, containing 422,672 samples in total. We randomly split the dataset into $70 \%$ training, $15 \%$ testing, and $15 \%$ validation sets.

Low-cost Features. We specify four tweet content related features to be low cost, since the attacker can trivially modify the content. They are, Mention Count, Hashtag Count, Tweet Count, and URL percent in tweets. All the other features are high cost, since they are related to the graph of redirection chains, which cannot be easily controlled by the attacker. Redirection chains form the traffic distribution systems in the underground economy, where different cybercriminals can purchase and re-sell the traffic [29, 55]. Thus graph-related features are largely outside the control of a single attacker, and are not trivial to change.

Monotonicity. Based on feature distribution measurement result, we specify that 7 shared resources-driven features are monotonically increasing, as shown in Table 3. Example measurement result is in Appendix C.2.

Stability. We specify low-cost features to be stable, with stable constant 8 . 


\begin{tabular}{|c|c|c|c|c|c|c|c|c|c|c|}
\hline \multirow[b]{2}{*}{ Model } & \multicolumn{5}{|c|}{ Performance } & \multicolumn{5}{|c|}{ Global Robustness Properties } \\
\hline & $\begin{array}{r}\text { TPR } \\
(\%) \\
\end{array}$ & $\begin{array}{r}\text { FPR } \\
(\%) \\
\end{array}$ & $\begin{array}{r}\text { Acc } \\
(\%) \\
\end{array}$ & AUC & $\mathrm{F} 1$ & Monotonicity & Stability & $\begin{array}{c}\text { High } \\
\text { Confidence }\end{array}$ & Redundancy & $\begin{array}{c}\text { Small } \\
\text { Neighborhood } \\
\end{array}$ \\
\hline \multicolumn{11}{|l|}{ Cryptojacking Detection } \\
\hline XGB & 100 & 0.3 & 99.8 & .99917 & .998 & $\bar{x}$ & $\bar{x}$ & $\checkmark$ & $\mathrm{N} / \mathrm{A}$ & $\bar{x}$ \\
\hline Neural Network & 100 & 0.2 & 99.9 & .99997 & .999 & $x$ & $x$ & $?$ & $\mathrm{~N} / \mathrm{A}$ & $x$ \\
\hline \multicolumn{11}{|l|}{ Models with Monotonicity Property } \\
\hline Monotonic XGB & 99.8 & 0.3 & 99.8 & .99969 & .998 & $\checkmark$ & $x$ & $\checkmark$ & $\mathrm{N} / \mathrm{A}$ & $x$ \\
\hline Nonnegative Linear & 97.7 & 0.2 & 98.8 & .99987 & .988 & $\checkmark$ & $x$ & $x$ & $\mathrm{~N} / \mathrm{A}$ & $x$ \\
\hline Nonnegative Neural Network & 99.7 & 0.2 & 99.8 & 99999 & .998 & $\checkmark$ & $x$ & $?$ & $\mathrm{~N} / \mathrm{A}$ & $\bar{x}$ \\
\hline Generalized UMNN & 99.8 & 0.2 & 99.8 & .99998 & .998 & $\boldsymbol{V}$ & $x$ & $?$ & $\mathrm{~N} / \mathrm{A}$ & $\bar{x}$ \\
\hline \multicolumn{11}{|c|}{ DL2 Models with Local Robustness Properties, trained using PGD attacks } \\
\hline DL2 Monotoncity & 99.7 & 0.2 & 99.8 & .99999 & .998 & $\bar{x}$ & $x$ & $?$ & $\mathrm{~N} / \mathrm{A}$ & $x$ \\
\hline DL2 Stability & 99.8 & 0.8 & 99.5 & 99987 & .995 & $\bar{x}$ & $x$ & $x$ & $\mathrm{~N} / \mathrm{A}$ & $\bar{x}$ \\
\hline DL2 High Confidence & 99.7 & 0.2 & 99.8 & 99999 & .998 & $x$ & $x$ & $?$ & $\mathrm{~N} / \mathrm{A}$ & $x$ \\
\hline DL2 Small Neighborhood & 99.8 & 0.3 & 99.8 & .99999 & .998 & $\bar{x}$ & $\bar{x}$ & $?$ & $\mathrm{~N} / \mathrm{A}$ & $\bar{x}$ \\
\hline DL2 Combined & 99.3 & 0.2 & 99.6 & .99985 & .996 & $\bar{x}$ & $\bar{x}$ & $x$ & $\mathrm{~N} / \mathrm{A}$ & $\bar{x}$ \\
\hline \multicolumn{11}{|c|}{ Our Models with Global Robustness Properties } \\
\hline Logic Ensemble Monotoncity & 100 & 0.3 & 99.8 & .99999 & .998 & $\checkmark$ & $x$ & $\checkmark$ & $\mathrm{N} / \mathrm{A}$ & $x$ \\
\hline Logic Ensemble Stability & 100 & 0.3 & 99.8 & .99831 & .998 & $x$ & $\checkmark$ & $\checkmark$ & $\mathrm{N} / \mathrm{A}$ & $\checkmark$ \\
\hline Logic Ensemble High Confidence & 100 & 0.3 & 99.8 & .99980 & .998 & $\bar{x}$ & $x$ & $\checkmark$ & $\mathrm{N} / \mathrm{A}$ & $\bar{x}$ \\
\hline Logic Ensemble Small Neighborhood & 100 & 0.3 & 99.8 & .99961 & .998 & $\bar{x}$ & $\checkmark$ & $\checkmark$ & $\mathrm{N} / \mathrm{A}$ & $\checkmark$ \\
\hline Logic Ensemble Combined & 100 & 3.2 & 98.4 & .99831 & .985 & $\checkmark$ & $\checkmark$ & $\checkmark$ & N/A & $\checkmark$ \\
\hline
\end{tabular}

Table 6: Results for training cryptojacking classifier with global robustness properties, compared to baseline models. N/A: property not specified. $\checkmark$ : verified to satisfy the property. $x$ : verified to not satisfy the property. ?: unknown.

High Confidence. We use a high confidence prediction threshold 0.98. Attacker is allowed to perturb any one of the low-cost features, but not multiple ones.

Small Neighborhood. We specify $\epsilon=1.5, c=10$, which means that the attacker can change each feature up to 1.5 times of its standard deviation, and the classifier output change is bounded by 15 .

\subsection{Baseline Models}

6.2.1 Experiment Setup. We compare against three types of baseline models, (1) tree ensemble and neural network that are not trained using any properties, (2) monotonic classifiers, and (3) neural network models trained with local robustness versions of our properties.

We train the following monotonic classifiers: monotonic gradient boosted decision trees using XGBoost (Monotonic XGB), linear classifier with nonnegative weights trained using logistic loss (Nonnegative Linear), nonnegative neural network, and generalized unconstrained monotonic neural network (UMNN) [89]. To evaluate against models with other properties, we train local versions of our properties using DL2 [25], which uses adversarial training. Malicious Class Gradient Weight. Since the Twitter spam account dataset [47] is missing some important features, we could not reproduce the exact model performance stated in the paper. Instead, we get $6 \%$ false positive rate. We contacted the authors but they don't have the missing data. Therefore, we tune the weight for the gradient of the malicious class in order to maintain low false positive rate for the models. We use line search tofi nd the best weight from 0.1 to 1 , which increments by 0.1 . Wefi nd that using 0.2 to weigh the gradient of the malicious class can keep the training false positive rate around $2 \%$ for this dataset. For the other two datasets, we do not weigh the gradients for different classes. Linear Classifier. The nonnegative linear classifier is a linear combination of input features with nonnegative weights, trained using logistic loss. If a feature is specified to be monotonically decreasing, we weigh the feature by -1 at input.

XGBoost Models. For the XGB model and Monotonic XGB model, we specify the following hyperparameters for three datasets. We use 4 boosting round, max depth 4 per tree to train the cryptojacking classifier, and 10 boosting rounds, max depth 5 to train Twitter spam account and Twitter spam URL classifiers.

Neural Network Models. The neural networks without any robustness properties as well as the nonnegative-weights networks have two fully connected layers, each with 200, 500, and 300 ReLU units for Cryptojacking, Twitter spam account, and Twitter Spam URL detection respectively. The generalized UMNNs, on the other hand, are positive linear combinations of multiple UMNN each with two fully connected layers and 50, 100, 100 ReLU nodes for each single monotonic feature.

We also use DL2 to train neural networks as baselines, which can achieve local robustness properties using adversarial training. All the DL2 models share the same architectures as the regular neural networks and the training objectives is to minimize the loss of PGD adversarial attacks [42] that target the robustness properties. We use 50 iterations with step sizes equal to one sixth of the allowable perturbation ranges for PGD attacks in the training process. For testing, we use the same PGD iterations and step sizes but with 10 random restarts.

For all the baseline neural networks mentioned above, we train 50 epochs to minimize binary cross-entropy loss on training datasets 
using Adam optimizer with learning rate 0.01 and piecewise learning rate scheduler.

6.2.2 Global Robustness Property Evaluation. To evaluate whether the baseline models have obtained global robustness properties, we use our Integer Linear Programming verifier to verify the XGB and linear models. For neural network models, we use PGD attacks to maximize the loss function for the property, as described in Section 6.2.1.

Table 6 and Table 8 show the results of evaluating global robustness properties for the baseline models. For neural network models, if the PGD attack has found counterexample for the property, we consider that the network does not satisfy the property. Otherwise, we use "?" to mark it as unknown/unverified.

Result 1: Monotonic XGB and generalized UMNN have the best true positive rate (TPR) among monotonic classifiers. For the two relatively large datasets, the performance of monotonic XGB and generalized UMNN are much better than nonnegativeweights models. For Twitter spam account detection, the TPR of monotonic XGB is $16.6 \%$ higher than the nonnegative linear classifier.

Result 2: Some baseline models naturally satisfy a few global robustness properties. The monotonic XGB model for crytpojacking detection satisfies the high confidence property, because it does not use the low-cost feature "hash function" in the tree structure. In comparison, our technique can train logic ensemble classifiers to satisfy the high confidence property but still use the low-cost feature to improve accuracy. Also, the nonnegative linear classifier for Twitter spam account detection satisfies the small neighborhood property, but it has only $70.1 \%$ TPR. Linear classifiers are known to be robust against small changes in input, however they have poor performance for many datasets.

Result 3: DL2 models cannot obtain global robustness. We found counterexamples for all DL2 models for Twitter spam URL detection, using PGD attacks over the property constraint loss, and most models trained with cryptojacking and Twitter spam account detection datasets. If the PGD attack fails tofi nd a counterexample, it does not mean that the model is verified to have the global property. There are always stronger attacks that mayfi nd counterexamples, as is often observed with adversarially trained models.

\subsection{Robust Logic Ensembles}

6.3.1 Training Algorithm Implementation. We implement our boosterfixer framework as the following. We use gradient boosting from XGBoost [10] as the booster. Within each round, we use the booster to add one tree to the existing classifier, and encode the classifier as the logic ensemble. This gives thefi xer the structure of clauses and weights $(\alpha, \beta, R)$ as the starting classifier with high accuracy.

To implement the verifier in thefi xer, we use APIs from Gurobi [1] to encode the integer linear program with boolean variables and property violation constraints, and then call the Gurobi solver to verify the global robustness properties of the logic ensemble. If the solver returns that the interger linear program is infeasible, the classifier is verified to satisfy the property. Otherwise, we construct a counterexample according to solutions for the boolean variables. For the trainer, we use PyTorch to implement the smoothed classifier as Continuous Logic Networks [72, 96]. Then, we use quadratic

\begin{tabular}{l|c}
\hline Dataset & Median Training Time \\
\hline \hline Cryptojacking & 25 min \\
\hline Twitter Spam Account & 29 hours \\
\hline Twitter Spam URL & 3 days \\
\hline
\end{tabular}

Table 7: Median training time for Logic Ensemble models.

programming to implement projected gradient descent. We compute the updated weights by minimizing the $\ell_{2}$ norm between the initial weights and the convex set defined by the training constraints. We implement the mini-batch training for the smoothed classifier, where we can specify the batch size. After one epoch of training, we discretize the classifier to the logic ensemble encoding for the verifier to verify the property again. We also implement a few heuristics to speed up the time for the verifier to generate counterexamples, with details described in Appendix E.

6.3.2 Experiment Setup. For the cryptojacking dataset, we boost 4 rounds, each adding a tree with max depth 4 . For the other two datasets, we boost 10 rounds, with max tree depth 5 , except that we only boost 6 rounds when training the Twitter spam account classifier with allfi ve properties. During CLN training, we keep track of the discrete classifier at each stage, including all the inequalities and conjunctions. When we need to smooth the classifier, we use shifted and scaled sigmoid function to smooth the inequality, with temperature $\frac{1}{500}$, shift by 0.01 , and product t-norm to smooth the conjunctions, to closely approximate the discrete classifier. The updated weights from gradient-guided training can be directly used for the discrete classifier. To discretize the model, we simply do not apply the sigmoid function and the product $t$-norm. We use the Adam optimizer with learning rate 0.001 and decay 0.95 , to minimize binary cross-entropy loss using gradient descent. For the crytpojacking dataset, we use mini-batch size 1 ; for the other two larger datasets, we use mini-batch size 1024. After boosting all the rounds, we choose the model with the highest validation AUC. Empirically, our algorithm converges well to an accurate classifier that satisfies the specified properties.

6.3.3 Global Robustness Property Evaluation. We train 15 logic ensemble models in total for the three datasets, each satisfying the specified global robustness properties, shown in Table 6 and Table 8 . We use our Integer Linear Program verifier (Section 4.2) to verify the properties for all models.

Training Overhead. Similar to most existing robust machine learning training strategies, training a verifiably robust model is significantly slower than training a non-robust model. We show the median training time for Logic Ensemble models in Table 7. Training non-robust XGBoost models takes one minute. However, computation is usually cheap and the tradeoff for getting more robustness in exchange for more computation is common across robust machine learning techniques. Next, we discuss our key results.

Result 4: Our monotonic models have comparable or better performance than existing methods. Our Logic Ensemble Monotonicity models have higher true positive rate and AUC than the Nonnegative Linear classifiers for all three datasets, and we also achieve better performance than the Nonnegative Neural Network models for the cryptojacking detection and Twitter account 


\begin{tabular}{|c|c|c|c|c|c|c|c|c|c|c|}
\hline \multirow[b]{2}{*}{ Model } & \multicolumn{5}{|c|}{ Performance } & \multicolumn{5}{|c|}{ Global Robustness Properties } \\
\hline & $\begin{array}{r}\text { TPR } \\
(\%) \\
\end{array}$ & $\begin{array}{r}\text { FPR } \\
(\%) \\
\end{array}$ & $\begin{array}{r}\text { Acc } \\
(\%) \\
\end{array}$ & AUC & $\mathrm{F} 1$ & Monotonicity & Stability & $\begin{array}{c}\text { High } \\
\text { Confidence }\end{array}$ & Redundancy & $\begin{array}{c}\text { Small } \\
\text { Neighborhood }\end{array}$ \\
\hline \multicolumn{11}{|l|}{ Twitter Spam Account Detection } \\
\hline $\mathrm{XGB}$ & 87.0 & 2.3 & 92.2 & .98978 & .920 & $x$ & $x$ & $x$ & $x$ & $x$ \\
\hline Neural Network & 86.4 & 2.5 & 91.8 & .98387 & .915 & $x$ & $x$ & $x$ & $x$ & $x$ \\
\hline \multicolumn{11}{|l|}{ Models with Monotonicity Property } \\
\hline Monotonic XGB & 86.7 & 2.7 & 91.9 & 98865 & .916 & $\boldsymbol{V}$ & $\bar{x}$ & $x$ & $\bar{x}$ & $x$ \\
\hline Nonnegative Linear & 70.1 & 2.4 & 83.5 & .95321 & .814 & $\checkmark$ & $x$ & $x$ & $x$ & $\checkmark$ \\
\hline Nonnegative Neural Network & 78.3 & 2.5 & 87.6 & .96723 & .867 & $\checkmark$ & $x$ & $x$ & $\bar{x}$ & $x$ \\
\hline Generalized UMNN & 86.0 & 3.9 & 90.9 & .97324 & .907 & $\checkmark$ & $x$ & $x$ & $\bar{x}$ & $x$ \\
\hline \multicolumn{11}{|c|}{ DL2 Models with Local Robustness Properties, trained using PGD attacks } \\
\hline DL2 Monotoncity & 83.2 & 2.6 & 90.1 & .97800 & .896 & $x$ & $x$ & $x$ & $x$ & $x$ \\
\hline DL2 Stability & 86.1 & 3.3 & 91.3 & .98029 & .910 & $x$ & $?$ & $x$ & $x$ & $x$ \\
\hline DL2 High Confidence & 82.8 & 2.6 & 89.9 & .98056 & .894 & $x$ & $x$ & $x$ & $x$ & $x$ \\
\hline DL2 Redundancy & 83.9 & 3.1 & 90.2 & .97898 & .898 & $x$ & $x$ & $?$ & $x$ & $x$ \\
\hline DL2 Small Neighborhood & 88.3 & 3.5 & 92.2 & .98086 & .921 & $x$ & $x$ & $x$ & $x$ & $x$ \\
\hline DL2 Combined & 83.8 & 3 & 90.2 & .97738 & .898 & $x$ & $x$ & $x$ & $x$ & $?$ \\
\hline \multicolumn{11}{|c|}{ Our Models with Global Robustness Properties } \\
\hline Logic Ensemble Monotoncity & 83.2 & 3.2 & 89.8 & .97297 & .894 & $\boldsymbol{V}$ & $\bar{x}$ & $\bar{x}$ & $\bar{x}$ & $x$ \\
\hline Logic Ensemble Stability & 86.0 & 2.1 & 91.8 & .98479 & .915 & $x$ & $\boldsymbol{V}$ & $x$ & $x$ & $x$ \\
\hline Logic Ensemble High Confidence & 86.1 & 2.6 & 91.6 & .98311 & .913 & $x$ & $\boldsymbol{V}$ & $\checkmark$ & $x$ & $x$ \\
\hline Logic Ensemble Redundancy & 85.5 & 3.2 & 91.0 & .98166 & .907 & $x$ & $\checkmark$ & $\boldsymbol{V}$ & $\checkmark$ & $x$ \\
\hline Logic Ensemble Small Neighborhood & 83.9 & 2.5 & 90.5 & .98325 & 0.901 & $x$ & $\checkmark$ & $x$ & $x$ & $\checkmark$ \\
\hline Logic Ensemble Combined & 81.6 & 2.4 & 89.4 & .98142 & .888 & $\checkmark$ & $\checkmark$ & $\boldsymbol{V}$ & $\checkmark$ & $\checkmark$ \\
\hline \multicolumn{11}{|l|}{ Twitter Spam URL Detection } \\
\hline XGB & 99.0 & 1.5 & 98.7 & .99834 & .986 & $x$ & $x$ & $x$ & N/A & $x$ \\
\hline Neural Network & 98.8 & 2.9 & 97.9 & .99735 & .977 & $x$ & $x$ & $x$ & N/A & $x$ \\
\hline \multicolumn{11}{|c|}{ Models with Monotonicity Property } \\
\hline Monotonic XGB & 99.4 & 1.7 & 98.8 & .99848 & .986 & $\checkmark$ & $x$ & $x$ & N/A & $x$ \\
\hline Nonnegative Linear & 93.2 & 18.6 & 86.7 & .90218 & .861 & $\checkmark$ & $x$ & $x$ & $\mathrm{~N} / \mathrm{A}$ & $x$ \\
\hline Nonnegative Neural Network & 98.0 & 6.9 & 95.3 & .98511 & .949 & $\checkmark$ & $x$ & $x$ & N/A & $x$ \\
\hline Generalized UMNN & 98.8 & 2.6 & 98.0 & .99732 & .977 & $\checkmark$ & $x$ & $x$ & $\mathrm{~N} / \mathrm{A}$ & $x$ \\
\hline \multicolumn{11}{|c|}{ DL2 Models with Local Robustness Properties, trained using PGD attacks } \\
\hline DL2 Monotoncity & 98.9 & 3.0 & 97.9 & .99694 & .976 & $x$ & $x$ & $x$ & $\mathrm{~N} / \mathrm{A}$ & $x$ \\
\hline DL2 Stability & 99.0 & 3.0 & 97.9 & .99706 & .977 & $x$ & $x$ & $x$ & $\mathrm{~N} / \mathrm{A}$ & $x$ \\
\hline DL2 High Confidence & 99.5 & 4.6 & 97.2 & .99696 & .969 & $x$ & $x$ & $x$ & N/A & $x$ \\
\hline DL2 Small Neighborhood & 99.1 & 3.0 & 97.9 & .99720 & .977 & $x$ & $x$ & $x$ & $\mathrm{~N} / \mathrm{A}$ & $x$ \\
\hline \multicolumn{11}{|c|}{ Our Models with Global Robustness Properties } \\
\hline Logic Ensemble Monotoncity & 96.3 & 3.5 & 96.4 & .98549 & .960 & $\checkmark$ & $x$ & $x$ & $\mathrm{~N} / \mathrm{A}$ & $x$ \\
\hline Logic Ensemble Stability & 92.9 & 3.3 & 95.0 & .98180 & .943 & $x$ & $\checkmark$ & $x$ & $\mathrm{~N} / \mathrm{A}$ & $\checkmark$ \\
\hline Logic Ensemble High Confidence & 97.6 & 5.4 & 95.9 & .98646 & .955 & $x$ & $\boldsymbol{V}$ & $\checkmark$ & $\mathrm{N} / \mathrm{A}$ & $x$ \\
\hline Logic Ensemble Small Neighborhood & 97.1 & 2.8 & 97.1 & .99338 & .968 & $x$ & $x$ & $x$ & $\mathrm{~N} / \mathrm{A}$ & $\checkmark$ \\
\hline
\end{tabular}

Table 8: Results for training Twitter account classifier and Twitter spam URL classifier with global robustness properties, compared to baseline models. N/A: property not specified. $\checkmark$ : verified to satisfy the property. $X$ : verified to not satisfy the property. ?: unknown.

detection datasets. Monotonic XGB outperforms our Logic Ensemble Monotonic models, but we still have comparable performance. For example, for the Twitter spam account detection, our Logic Ensemble Monotonicity model has 3.5\% lower true positive rate (TPR), and $0.5 \%$ higher false positive rate (FPR) than the Monotonic XGB model.

Result 5: Our models have moderate performance drop to obtain an individual property. For cryptojacking detection, enforcing each property does not decrease TPR at all, and only increases FPR by $0.1 \%$ compared to the baseline neural network model (Table 6). For Twitter spam account detection, logic ensemble models that satisfy one global robustness property decrease the TPR by at most $3.8 \%$, and increase the FPR by at most $0.9 \%$, compared to the baseline XGB model (Table 8). For Twitter spam URL detection, within monotonicity, stability, and small neighborhood properties, enforcing one property for the classifier can maintain high TPR (from $92.9 \%$ to $97.6 \%$ ) and low FPR (from $2.8 \%$ to $5.4 \%$, Table 8). For example, the Logic Ensemble High Confidence model decreases the TPR by $1.4 \%$ and increases the FPR by $3.9 \%$, compared to the baseline XGB model. This model utilizes the low-cost features to improve the 
prediction accuracy. If we only use high-cost features to train a tree ensemble with the same capacity (10 rounds of boosting), we can only achieve $79.9 \%$ TPR and 0.96075 AUC. In comparison, our Logic Ensemble High Confidence model has 97.6\% TPR and 0.98646 AUC. Results regarding hyperparameters are discussed in Appendix F.

Result 6: Training a classifier with one property sometimes obtains another property. Table 6 shows that all cryptojacking Logic Ensemble classifiers that were enforced with only one property, have obtained at least one other property. For example, the Logic Ensemble Stability model has obtained small neighborhood property, and vice versa. Since we specify all features to be stable for this dataset, the stability property is equivalent to the global Lipschitz property under $L_{0}$ distance. On the other hand, we define the small neighborhood property with a new distance. This shows that enforcing robustness for one property can generalize the robustness to a different property. More results are discussed in Appendix G.

Result 7: We can train classifiers to satisfy multiple global robustness properties at the same time. We train a cryptojacking classifier with four properties, and a Twitter spam account classifier withfi ve properties. For cryptojacking detection, the Logic Ensemble Combined model maintains the same high TPR, and only increases the FPR by 3\% compared to the baseline neural network model (Table 6). For Twitter spam account detection, the Logic Ensemble Combined model that satisfies all properties only decreases the TPR by $5.4 \%$ and increases the FPR by $0.1 \%$, compared to the baseline XGB model with no property (Table 8 ). More results are discussed in Appendix $\mathrm{H}$.

\section{RELATED WORK}

Program Synthesis. Solar-Lezama et al. [80] proposed counterexample guided inductive synthesis (CEGIS) to synthesizefi nite programs according to specifications of desired functionalities. The key idea is to iteratively generate a proposal of the program and check the correctness of the program, where the checker should be able to generate counterexamples of correctness to guide the program generation process. The general idea of CEGIS has also been used to learn recursive logic programs (e.g., as static analysis rules) [2, 15, $69,75]$. We design ourfi xer following the general form of CEGIS.

Local Robustness. Many techniques have been proposed to verify local robustness (e.g., $\ell_{p}$ robustness) of neural networks, including customized solvers [34, 38, 39, 83] and bound propagation based verification methods $[6,8,53,63,64,71,74,76-78,86-$ $88,90,92,94,98]$. Bound propagation verifiers can also be applied in robust optimization to train the models with certified local robustness [9, 11, 54, 62, 85, 93, 97, 99]. Randomized smoothing $[14,36,45,52,73,95]$ is another technique to provide probabilistic local robustness guarantee. Several methods have been proposed to utilize the local Lipshitz constant of neural networks for verification [33, 90, 91], and constrain or use the local Lipshitz bounds to train robust networks [4, 12, 13, 22, 24, 30, 49, 65, 68, 79, 81].

Global Robustness. Fischer et al. [25] and Melacci et al. [60] proposed global robustness properties for image classifiers using universally quantified statements. Both of their techniques smooth the logic expression of the property into a differentiable loss function, and then use PGD attacks [42] to minimize the loss. They can train neural networks to obtain local robustness, but cannot obtain verified global robustness. ART [56] proposed an abstraction refinement strategy to train provably correct neural networks. The model satisfies global robustness properties when the correctness loss reaches zero. However, in practice their correctness loss did not converge to zero. Leino et al. [50] proposed to minimize global Lipschitz constant to train globally-robust neural networks, but they can only verify one global property that abstains on non-robust predictions. Monotonic Classifiers. Many methods have been proposed to train monotonic classifiers [5, 7, 16, 17, 23, 32, 35, 40, 89]. Recently, Wehenkel et al. [89] proposed unconstrained monotonic neural networks, based on the key idea that a function is monotonic as long as its derivative is nonnegative. This has increased the performance of monotonic neural network significantly compared to enforcing nonnegative weights. Incer et al. [35] used monotone constraints from XGBoost to train monotonic malware classifiers. XGBoost enforces monotone constraints for the left child weight to be always smaller (or greater) than the right child, which is a specialized method and does not generalize to other global robustness properties.

Discrete Classifier and Smoothing. Friedman et al. [27] proposed rule ensemble, where the each rule is a path in the decision tree, and they used regression to learn how to combine rules. Our logic ensemble is more general such that the clauses do not have to form a tree structure. We only take rules from trees as the starting classifier tofi $x$ the properties. Kantchelian et al. [37] proposed the mixed integer linear program attack to evade tree ensembles by perturbing a concrete input. In comparison, our integer linear program verifier has only integer variables, and represents all inputs symbolically. Continuous Logic Networks was proposed to smooth SMT formulas to learn loop invariants $[72,96]$. In this paper, we apply the smoothing techniques to train machine learning classifiers.

\section{CONCLUSION}

In this paper, we have presented a novel booster-fixer training framework to enforce new global robustness properties for security classifiers. We have formally defined six global robustness properties, of whichfi ve are new. Our training technique is general, and can handle a large class of properties. We have used experiments to show that we can train different security classifiers to satisfy multiple global robustness properties at the same time.

\section{ACKNOWLEDGEMENTS}

We thank the anonymous reviewers for their constructive and valuable feedback. This work is supported in part by NSF grants CNS18-50725, CCF-21-24225; generous gifts from Open Philanthropy, two Google Faculty Fellowships, Berkeley Artificial Intelligence Research (BAIR), a Capital One Research Grant, a J.P. Morgan Faculty Award; and Institute of Information \& communications Technology Planning \& Evaluation (IITP) grant funded by the Korea government(MSIT) (No.2020-0-00153). Any opinions,fi ndings, conclusions, or recommendations expressed herein are those of the authors, and do not necessarily reflect those of the US Government, NSF, Google, Capital One, J.P. Morgan, or the Korea government.

\section{REFERENCES}

[1] [n.d.]. Gurobi Optimization. https://www.gurobi.com/. 
[2] Aws Albarghouthi, Paraschos Koutris, Mayur Naik, and Calvin Smith. 2017. Constraint-based synthesis of Datalog programs. In International Conference on Principles and Practice of Constraint Programming. Springer, 689-706.

[3] Kevin Allix, Tegawendé F Bissyandé, Jacques Klein, and Yves Le Traon. 2015. Are your training datasets yet relevant?. In International Symposium on Engineering Secure Software and Systems. Springer, 51-67.

[4] Cem Anil, James Lucas, and Roger Grosse. 2019. Sorting out Lipschitz function approximation. In International Conference on Machine Learning. PMLR, 291-301.

[5] Norman P Archer and Shouhong Wang. 1993. Application of the back propagation neural network algorithm with monotonicity constraints for two-group classification problems. Decision Sciences 24, 1 (1993), 60-75.

[6] Mislav Balunović, Maximilian Baader, Gagandeep Singh, Timon Gehr, and Martin Vechev. 2019. Certifying geometric robustness of neural networks. Advances in Neural Information Processing Systems (NeurIPS) (2019).

[7] Arie Ben-David. 1995. Monotonicity maintenance in information-theoretic machine learning algorithms. Machine Learning 19, 1 (1995), 29-43.

[8] Akhilan Boopathy, Tsui-Wei Weng, Pin-Yu Chen, Sijia Liu, and Luca Daniel. 2019. Cnn-cert: An efficient framework for certifying robustness of convolutional neural networks. In AAAI Conference on Artificial Intelligence (AAAI).

[9] Akhilan Boopathy, Tsui-Wei Weng, Sijia Liu, Pin-Yu Chen, Gaoyuan Zhang, and Luca Daniel. 2021. Fast Training of Provably Robust Neural Networks by SingleProp. AAAI Conference on Artificial Intelligence (AAAI) (2021).

[10] Tianqi Chen and Carlos Guestrin. 2016. Xgboost: A scalable tree boosting system. In Proceedings of the 22nd acm sigkdd international conference on knowledge discovery and data mining. ACM, 785-794.

[11] Yizheng Chen, Shiqi Wang, Dongdong She, and Suman Jana. 2020. On Training Robust PDF Malware Classifiers. In USENIX Security Symposium.

[12] Moustapha Cisse, Piotr Bojanowski, Edouard Grave, Yann Dauphin, and Nicolas Usunier. 2017. Parseval networks: Improving robustness to adversarial examples. In International Conference on Machine Learning. PMLR, 854-863.

[13] Jeremy EJ Cohen, Todd Huster, and Ra Cohen. 2019. Universal lipschitz approximation in bounded depth neural networks. arXiv preprint arXiv:1904.04861(2019).

[14] Jeremy M Cohen, Elan Rosenfeld, and J Zico Kolter. 2019. Certified adversarial robustness via randomized smoothing. International Conference on Machine Learning (2019)

[15] Andrew Cropper, Sebastijan Dumančić, and Stephen H Muggleton. 2020. Turning 30: New ideas in inductive logic programming. In International foint Conferences on Artifical Intelligence (IFCAI).

[16] Hennie Daniels and Marina Velikova. 2010. Monotone and partially monotone neural networks. IEEE Transactions on Neural Networks 21, 6 (2010), 906-917.

[17] Wouter Duivesteijn and Ad Feelders. 2008. Nearest neighbour classification with monotonicity constraints. In foint European Conference on Machine Learning and Knowledge Discovery in Databases. Springer, 301-316.

[18] Souradeep Dutta, Susmit Jha, Sriram Sankaranarayanan, and Ashish Tiwari. 2018. Output Range Analysis for Deep Feedforward Neural Networks. In NASA Formal Methods Symposium. Springer, 121-138.

[19] Krishnamurthy Dvijotham, Sven Gowal, Robert Stanforth, Relja Arandjelovic Brendan O'Donoghue, Jonathan Uesato, and Pushmeet Kohli. 2018. Training verified learners with learned verifiers. arXiv preprint arXiv:1805.10265 (2018).

[20] Krishnamurthy Dvijotham, Robert Stanforth, Sven Gowal, Timothy Mann, and Pushmeet Kohli. 2018. A dual approach to scalable verification of deep networks. arXiv preprint arXiv:1803.06567 (2018).

[21] Ruediger Ehlers. 2017. Formal Verification of Piece-Wise Linear Feed-Forward Neural Networks. 15th International Symposium on Automated Technology for Verification and Analysis (2017).

[22] Farzan Farnia, Jesse Zhang, and David Tse. 2018. Generalizable Adversarial Training via Spectral Normalization. In International Conference on Learning Representations.

[23] Ad Feelders. 2010. Monotone relabeling in ordinal classification. In 2010 IEEE International Conference on Data Mining. IEEE, 803-808.

[24] Chris Finlay and Adam M Oberman. 2021. Scaleable input gradient regularization for adversarial robustness. Machine Learning with Applications 3 (2021), 100017.

[25] Marc Fischer, Mislav Balunovic, Dana Drachsler-Cohen, Timon Gehr, Ce Zhang, and Martin Vechev. 2019. DL2: Training and Querying Neural Networks with Logic. In International Conference on Machine Learning (ICML).

[26] Matteo Fischetti and Jason Jo. 2017. Deep Neural Networks as 0-1 Mixed Integer Linear Programs: A Feasibility Study. arXiv preprint arXiv:1712.06174 (2017).

[27] Jerome H Friedman, Bogdan E Popescu, et al. 2008. Predictive learning via rule ensembles. The Annals of Applied Statistics 2, 3 (2008), 916-954.

[28] Timon Gehr, Matthew Mirman, Dana Drachsler-Cohen, Petar Tsankov, Swarat Chaudhuri, and Martin Vechev. 2018. Ai 2: Safety and robustness certification of neural networks with abstract interpretation. In IEEE Symposium on Security and Privacy (SP).

[29] Maxim Goncharov. [n.d.]. Traffic direction systems as malware distribution tools http://www.trendmicro.es/media/misc/malware-distribution-tools-researchpaper-en.pdf.

[30] Henry Gouk, Eibe Frank, Bernhard Pfahringer, and Michael J Cree. 2021 Regularisation of neural networks by enforcing lipschitz continuity. Machine
Learning 110, 2 (2021), 393-416.

[31] Kathrin Grosse, Nicolas Papernot, Praveen Manoharan, Michael Backes, and Patrick McDaniel. 2016. Adversarial perturbations against deep neural networks for malware classification. arXiv preprint arXiv:1606.04435 (2016)

[32] Maya Gupta, Andrew Cotter, Jan Pfeifer, Konstantin Voevodski, Kevin Canini, Alexander Mangylov, Wojciech Moczydlowski, and Alexander Van Esbroeck. 2016. Monotonic calibrated interpolated look-up tables. The fournal of Machine Learning Research 17, 1 (2016), 3790-3836.

[33] Matthias Hein and Maksym Andriushchenko. 2017. Formal guarantees on the robustness of a classifier against adversarial manipulation. In Advances in Neural Information Processing Systems.

[34] Xiaowei Huang, Marta Kwiatkowska, Sen Wang, and Min Wu. 2017. Safety verification of deep neural networks. In International Conference on Computer Aided Verification (CAV). Springer, 3-29.

[35] Inigo Incer, Michael Theodorides, Sadia Afroz, and David Wagner. 2018. Adversarially Robust Malware Detection Using Monotonic Classification. In Proceedings of the Fourth ACM International Workshop on Security and Privacy Analytics. ACM, 54-63.

[36] Jinyuan Jia, Xiaoyu Cao, Binghui Wang, and Neil Zhenqiang Gong. 2019. Certified robustness for top-k predictions against adversarial perturbations via randomized smoothing. International Conference on Learning Representations (ICLR) (2019).

[37] Alex Kantchelian, JD Tygar, and Anthony Joseph. 2016. Evasion and hardening of tree ensemble classifiers. In International Conference on Machine Learning. 2387-2396.

[38] Guy Katz, Clark Barrett, David L Dill, Kyle Julian, and Mykel J Kochenderfer. 2017. Reluplex: An efficient SMT solver for verifying deep neural networks. In International Conference on Computer Aided Verification (CAV). Springer, 97-117.

[39] Guy Katz, Derek A Huang, Duligur Ibeling, Kyle Julian, Christopher Lazarus, Rachel Lim, Parth Shah, Shantanu Thakoor, Haoze Wu, Aleksandar Zeljić, et al. 2019. The marabou framework for verification and analysis of deep neural networks. In International Conference on Computer Aided Verification (CAV).

[40] Herbert Kay and Lyle H Ungar. 2000. Estimating monotonic functions and their bounds. AIChE fournal 46, 12 (2000), 2426-2434.

[41] Amin Kharraz, Zane Ma, Paul Murley, Charles Lever, Joshua Mason, Andrew Miller, Nikita Borisov, Manos Antonakakis, and Michael Bailey. 2019. Outguard: Detecting in-browser covert cryptocurrency mining in the wild. In The World Wide Web Conference. 840-852.

[42] Alexey Kurakin, Ian Goodfellow, and Samy Bengio. 2017. Adversarial machine learning at scale. In International Conference on Learning Representations (ICLR).

[43] Heeyoung Kwon, Mirza Basim Baig, and Leman Akoglu. 2017. A domain-agnostic approach to spam-URL detection via redirects. In Pacific-Asia Conference on Knowledge Discovery and Data Mining. Springer, 220-232.

[44] Pavel Laskov et al. 2014. Practical evasion of a learning-based classifier: A case study. In Security and Privacy (SP), 2014 IEEE Symposium on. IEEE, 197-211.

[45] Mathias Lecuyer, Vaggelis Atlidakis, Roxana Geambasu, Daniel Hsu, and Suman Jana. 2019. Certified robustness to adversarial examples with differential privacy. In 2019 IEEE Symposium on Security and Privacy (SP). IEEE.

[46] Kyumin Lee, James Caverlee, and Steve Webb. 2010. Uncovering social spammers: social honeypots + machine learning. In Proceedings of the 33rd international ACM SIGIR conference on Research and development in information retrieval. 435-442.

[47] Kyumin Lee, Brian Eoff, and James Caverlee. 2011. Seven months with the devils: A long-term study of content polluters on twitter. In Proceedings of the International AAAI Conference on Web and Social Media, Vol. 5.

[48] Sangho Lee and Jong Kim. 2013. Warningbird: A near real-time detection system for suspicious urls in twitter stream. IEEE transactions on dependable and secure computing 10, 3 (2013), 183-195.

[49] Sungyoon Lee, Jaewook Lee, and Saerom Park. 2020. Lipschitz-Certifiable Training with a Tight Outer Bound. Advances in Neural Information Processing Systems (NeurIPS) (2020).

[50] Klas Leino, Zifan Wang, and Matt Fredrikson. 2021. Globally-Robust Neural Networks. arXiv preprint arXiv:2102.08452 (2021).

[51] Bai Li, Changyou Chen, Wenlin Wang, and Lawrence Carin. 2018. Second-order adversarial attack and certifiable robustness. (2018).

[52] Bai Li, Changyou Chen, Wenlin Wang, and Lawrence Carin. 2019. Certified adversarial robustness with additive noise. Advances in Neural Information Processing Systems (NeurIPS) (2019).

[53] Linyi Li, Xiangyu Qi, Tao Xie, and Bo Li. 2020. SoK: Certified Robustness for Deep Neural Networks. arXiv preprint arXiv:2009.04131 (2020).

[54] Linyi Li, Zexuan Zhong, Bo Li, and Tao Xie. 2019. Robustra: Training Provable Robust Neural Networks over Reference Adversarial Space. In IfCAI.

[55] Zhou Li, Sumayah Alrwais, Yinglian Xie, Fang Yu, and XiaoFeng Wang. 2013. Finding the linchpins of the dark web: a study on topologically dedicated hosts on malicious web infrastructures. In 2013 IEEE Symposium on Security and Privacy. IEEE, 112-126.

[56] Xuankang Lin, He Zhu, Roopsha Samanta, and Suresh Jagannathan. 2020. ART: abstraction refinement-guided training for provably correct neural networks. In 2020 Formal Methods in Computer Aided Design (FMCAD). IEEE, 148-157. 
[57] Alessio Lomuscio and Lalit Maganti. 2017. An approach to reachability analysis for feed-forward relu neural networks. arXiv preprint arXiv:1706.07351 (2017).

[58] Justin Ma, Lawrence K Saul, Stefan Savage, and Geoffrey M Voelker. 2009. Identifying suspicious URLs: an application of large-scale online learning. In Proceedings of the 26th annual international conference on machine learning. 681-688.

[59] Aleksander Madry, Aleksandar Makelov, Ludwig Schmidt, Dimitris Tsipras, and Adrian Vladu. 2018. Towards deep learning models resistant to adversarial attacks. International Conference on Learning Representations (ICLR) (2018).

[60] Stefano Melacci, Gabriele Ciravegna, Angelo Sotgiu, Ambra Demontis, Battista Biggio, Marco Gori, and Fabio Roli. 2020. Can Domain Knowledge Alleviate Adversarial Attacks in Multi-Label Classifiers? arXiv preprint arXiv:2006.03833 (2020).

[61] Brad Miller, Alex Kantchelian, Michael Carl Tschantz, Sadia Afroz, Rekha Bachwani, Riyaz Faizullabhoy, Ling Huang, Vaishaal Shankar, Tony Wu, George Yiu, et al. 2016. Reviewer integration and performance measurement for malware detection. In International Conference on Detection of Intrusions and Malware, and Vulnerability Assessment. Springer, 122-141.

[62] Matthew Mirman, Timon Gehr, and Martin Vechev. 2018. Differentiable Abstract Interpretation for Provably Robust Neural Networks. In International Conference on Machine Learning (ICML). 3575-3583.

[63] Christoph Müller, François Serre, Gagandeep Singh, Markus Püschel, and Martin Vechev. 2021. Scaling Polyhedral Neural Network Verification on GPUs. Proceedings of Machine Learning and Systems 3 (2021).

[64] Mark Niklas Müller, Gleb Makarchuk, Gagandeep Singh, Markus Püschel, and Martin Vechev. 2021. Precise Multi-Neuron Abstractions for Neural Network Certification. arXiv preprint arXiv:2103.03638 (2021).

[65] Patricia Pauli, Anne Koch, Julian Berberich, Paul Kohler, and Frank Allgower 2021. Training robust neural networks using Lipschitz bounds. IEEE Control Systems Letters (2021)

[66] Feargus Pendlebury, Fabio Pierazzi, Roberto Jordaney, Johannes Kinder, and Lorenzo Cavallaro. 2019. TESSERACT: Eliminating experimental bias in malware classification across space and time. In 28th USENIX Security Symposium (USENIX Security 19). 729-746.

[67] F. Pierazzi, F. Pendlebury, J. Cortellazzi, and L. Cavallaro. 2020 Intriguing Properties of Adversarial ML Attacks in the Problem Space. In 2020 IEEE Symposium on Security and Privacy (SP). IEEE Computer Society, 1308-1325. https://doi.org/10.1109/SP40000.2020.00073

[68] Chongli Qin, James Martens, Sven Gowal, Dilip Krishnan, Krishnamurthy Dvijotham, Alhussein Fawzi, Soham De, Robert Stanforth, and Pushmeet Kohli. 2019. Adversarial robustness through local linearization. Advances in Neural Information Processing Systems (NIPS) (2019).

[69] Mukund Raghothaman, Jonathan Mendelson, David Zhao, Mayur Naik, and Bernhard Scholz. 2019. Provenance-guided synthesis of Datalog programs. Proceedings of the ACM on Programming Languages 4, POPL (2019), 1-27.

[70] Aditi Raghunathan, Jacob Steinhardt, and Percy Liang. 2018. Certified defenses against adversarial examples. International Conference on Learning Representations (ICLR) (2018).

[71] Aditi Raghunathan, Jacob Steinhardt, and Percy S Liang. 2018. Semidefinite relaxations for certifying robustness to adversarial examples. In Advances in Neural Information Processing Systems. 10900-10910.

[72] Gabriel Ryan, Justin Wong, Jianan Yao, Ronghui Gu, and Suman Jana. 2020. CLN2INV: Learning Loop Invariants with Continuous Logic Networks. In International Conference on Learning Representations (ICLR).

[73] Hadi Salman, Greg Yang, Jerry Li, Pengchuan Zhang, Huan Zhang, Ilya Razenshteyn, and Sebastien Bubeck. 2019. Provably robust deep learning via adversarially trained smoothed classifiers. Advances in Neural Information Processing Systems (NeurIPS) (2019).

[74] Hadi Salman, Greg Yang, Huan Zhang, Cho-Jui Hsieh, and Pengchuan Zhang 2019. A convex relaxation barrier to tight robustness verification of neural networks. Advances in Neural Information Processing Systems (NeurIPS) (2019).

[75] Xujie Si, Woosuk Lee, Richard Zhang, Aws Albarghouthi, Paraschos Koutris, and Mayur Naik. 2018. Syntax-guided synthesis of datalog programs. In Proceedings of the 2018 26th ACM Foint Meeting on European Software Engineering Conference and Symposium on the Foundations of Software Engineering. 515-527.

[76] Gagandeep Singh, Rupanshu Ganvir, Markus Püschel, and Martin Vechev. 2019. Beyond the single neuron convex barrier for neural network certification. Advances in Neural Information Processing Systems (NeurIPS) (2019).

[77] Gagandeep Singh, Timon Gehr, Markus Püschel, and Martin Vechev. 2019. An abstract domain for certifying neural networks. Proceedings of the ACM on Programming Languages 3, POPL (2019), 1-30.

[78] Gagandeep Singh, Timon Gehr, Markus Püschel, and Martin T Vechev. 2019. Boosting Robustness Certification of Neural Networks.. In ICLR (Poster).

[79] Sahil Singla and Soheil Feizi. 2019. Bounding singular values of convolution layers. arXiv preprint arXiv:1911.10258 (2019).

[80] Armando Solar-Lezama, Liviu Tancau, Rastislav Bodik, Sanjit Seshia, and Vijay Saraswat. 2006. Combinatorial sketching forfi nite programs. In Proceedings of the 12th international conference on Architectural support for programming languages and operating systems. 404-415.

[81] Christian Szegedy, Wojciech Zaremba, Ilya Sutskever, Joan Bruna, Dumitru Erhan, Ian Goodfellow, and Rob Fergus. 2013. Intriguing properties of neural networks. International Conference on Learning Representations (ICLR) (2013).

[82] Kurt Thomas, Chris Grier, Justin Ma, Vern Paxson, and Dawn Song. 2011. Design and evaluation of a real-time url spamfi ltering service. In 2011 IEEE symposium on security and privacy. IEEE, 447-462.

[83] Vincent Tjeng, Kai Xiao, and Russ Tedrake. 2017. Evaluating Robustness of Neural Networks with Mixed Integer Programming. arXiv preprint arXiv:1711.07356 (2017).

[84] David Wagner and Paolo Soto. 2002. Mimicry attacks on host-based intrusion detection systems. In Proceedings of the 9th ACM Conference on Computer and Communications Security. ACM, 255-264.

[85] Shiqi Wang, Yizheng Chen, Ahmed Abdou, and Suman Jana. 2018. MixTrain: Scalable Training of Formally Robust Neural Networks. arXiv preprint arXiv:1811.02625 (2018).

[86] Shiqi Wang, Kexin Pei, Whitehouse Justin, Junfeng Yang, and Suman Jana. 2018. Efficient Formal Safety Analysis of Neural Networks. Advances in Neural Information Processing Systems (NIPS) (2018).

[87] Shiqi Wang, Kexin Pei, Whitehouse Justin, Junfeng Yang, and Suman Jana. 2018. Formal Security Analysis of Neural Networks using Symbolic Intervals. 27th USENIX Security Symposium (2018).

[88] Shiqi Wang, Huan Zhang, Kaidi Xu, Xue Lin, Suman Jana, Cho-Jui Hsieh, and J Zico Kolter. 2021. Beta-CROWN: Efficient Bound Propagation with Per-neuron Split Constraints for Complete and Incomplete Neural Network Verification. arXiv preprint arXiv:2103.06624 (2021).

[89] Antoine Wehenkel and Gilles Louppe. 2019. Unconstrained monotonic neural networks. In Advances in Neural Information Processing Systems.

[90] Lily Weng, Huan Zhang, Hongge Chen, Zhao Song, Cho-Jui Hsieh, Luca Daniel, Duane Boning, and Inderjit Dhillon. 2018. Towards fast computation of certified robustness for relu networks. In International Conference on Machine Learning (ICML).

[91] Tsui-Wei Weng, Huan Zhang, Pin-Yu Chen, Jinfeng Yi, Dong Su, Yupeng Gao, Cho-Jui Hsieh, and Luca Daniel. 2018. Evaluating the robustness of neural networks: An extreme value theory approach. In International Conference on Learning Representations (ICLR).

[92] Eric Wong and Zico Kolter. 2018. Provable defenses against adversarial examples via the convex outer adversarial polytope. In International Conference on Machine Learning. 5283-5292.

[93] Eric Wong, Frank Schmidt, Jan Hendrik Metzen, and J Zico Kolter. 2018. Scaling provable adversarial defenses. Advances in Neural Information Processing Systems (NIPS) (2018).

[94] Kaidi Xu, Huan Zhang, Shiqi Wang, Yihan Wang, Suman Jana, Xue Lin, and Cho-Jui Hsieh. 2021. Fast and complete: Enabling complete neural network verification with rapid and massively parallel incomplete verifiers. International Conference on Learning Representations (ICLR) (2021).

[95] Greg Yang, Tony Duan, J Edward Hu, Hadi Salman, Ilya Razenshteyn, and Jerry Li. 2020. Randomized smoothing of all shapes and sizes. In International Conference on Machine Learning (ICML). PMLR.

[96] Jianan Yao, Gabriel Ryan, Justin Wong, Suman Jana, and Ronghui Gu. 2020. Learning Nonlinear Loop Invariants with Gated Continuous Logic Networks. In Proceedings of the 41st ACM SIGPLAN Conference on Programming Language Design and Implementation.

[97] Huan Zhang, Hongge Chen, Chaowei Xiao, Sven Gowal, Robert Stanforth, Bo Li, Duane Boning, and Cho-Jui Hsieh. 2020. Towards stable and efficient training of verifiably robust neural networks. International Conference on Learning Representations (ICLR) (2020).

[98] Huan Zhang, Tsui-Wei Weng, Pin-Yu Chen, Cho-Jui Hsieh, and Luca Daniel. 2018. Efficient neural network robustness certification with general activation functions. arXiv preprint arXiv:1811.00866 (2018).

[99] Xiao Zhang and David Evans. 2019. Cost-Sensitive Robustness against Adversarial Examples. International Conference on Learning Representations (ICLR) (2019).

\section{A STABILITY FOR TWITTER ACCOUNT CLASSIFIER}

To classify Twitter accounts that broadcast spam URLs, we can use the number of followers and the ratio of posted URLs over total number of tweets as features [47]. It is hard for spammers to obtain large amount of followers, and they are likely to post more URLs than benign users. We specify the URLRatio feature to be stable, such that arbitrarily changing the feature will not change the classifier's output by more than 1 .

Figure 4 shows one CEGIS iteration to train the stability property. The starting classifier is a decision tree. For example, "1.0 * URLRatio $<0.285 \wedge 1.0 *$ followers $<1429.5 \rightarrow-1.71$ ” means 


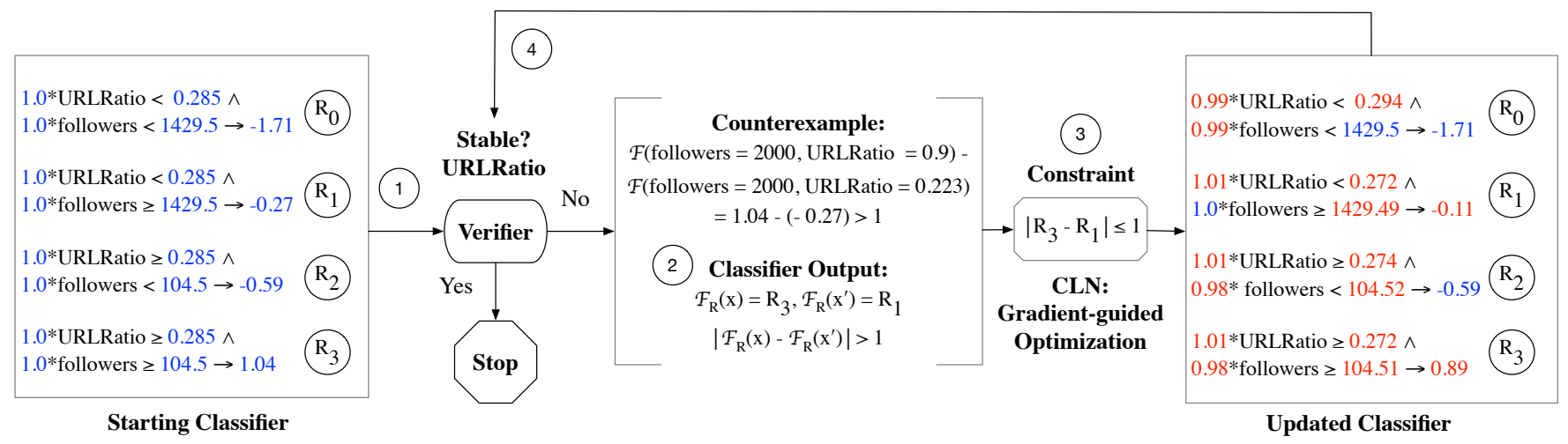

Figure 4: One CEGIS iteration to train stability property for the Twitter account classifier. We specify the classifier's output score to change at most by one when the URLRatio feature is arbitrarily perturbed. Multiple weights of the classifiers are updated by gradient-guided optimization, and the classifier after training no longer forms a tree structure.

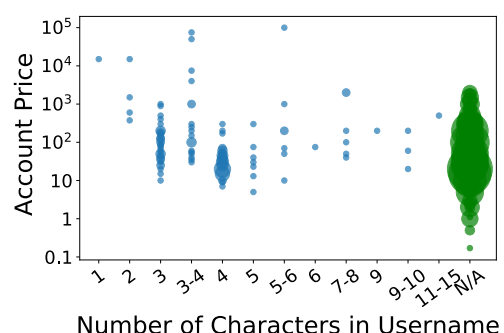

Figure 5: Price (\$, log scale) of Twitter accounts with different number of characters.

that if the URLRatio and the number of followers both satisfy these inequalities, the clause is true and returns -1.71 , value of the variable $R_{0}$. Otherwise, the clause returns 0 . We take the sum of return values from all clauses to be the classification score. One CEGIS iteration goes through the following four steps.

Step (1): We ask the verifier whether the URLRatio feature is stable. If the verifier can verify the stability property, we stop here. If not, the verifier generates a counterexample that violates the property. Here, the counterexample shows that if the number of followers is 2000, and if the URLRatio feature changes from 0.9 to 0.223 , the classifier's output changes by 1.31 , which violates the stability property.

Step (2): Using the sum of true clauses for each input, we represent $x, x^{\prime}$ as $\mathcal{F}_{R}(x)=R_{3}$, and $\mathcal{F}_{R}\left(x^{\prime}\right)=R_{1}$.

Step (3): We construct the constraint to eliminate the counterexample. In this case, we want the difference between the output for $x$ and the output for $x^{\prime}$ to be bounded by 1 , i.e., $\left|R_{3}-R_{1}\right| \leq 1$. Then, we smooth the classifier using CLN $[72,96]$, train the weights using projected gradient descent with the constraint. After one epoch, we have updated the classifier in the rightmost box of Figure 4. The red weights of the model are updated by gradient descent. Note that the classifier no longer follows a tree structure.

Lastly, we repeat this process until the classifier is verified to satisfy the property (Step (4)). In this example, the updated classifier still does not satisfy stability, and we will go through more CEGIS iterations to update it.

\section{B PROOF}

Lemma 1. If a classifier satisfies Property 3a, then it also satisfies Property 3.

Proof. $\forall x, x^{\prime} \in \mathbb{R}^{n} .\left[\forall i \notin J . x_{i}=x_{i}^{\prime}\right] \wedge g(\mathcal{F}(x)) \geq \delta$, we have $\mathcal{F}(x) \geq g^{-1}(\delta)$. Since $\mathcal{F}$ satisfies Property 3 a, then we also have $\mathcal{F}(x)-\mathcal{F}\left(x^{\prime}\right) \leq g^{-1}(\delta)$. Therefore, $\mathcal{F}\left(x^{\prime}\right) \geq \mathcal{F}(x)-g^{-1}(\delta) \geq 0$.

\section{MEASUREMENT RESULTS}

\section{C.1 LenScreenName Feature}

We measure the economic cost for attackers to perturb the LenScreenName feature from the Twitter spam account dataset. We extract the number of characters information from 6,125 for-sale Twitter account posts, and measure the price for accounts with different username length. or unspecificed characters. In Figure 5, we plot the price of accounts according to the username length. If the post says " 3 or 4 characters", we plot the price under " $3-4$ " category. The majority of accounts are under "N/A" category, where the sellers do not mention the length of username, but emphasize other attributes such as number of followers. Overall, if the username length has at most 4 characters, it affects the account price more than longer username.

\section{C.2 CCSize Feature}

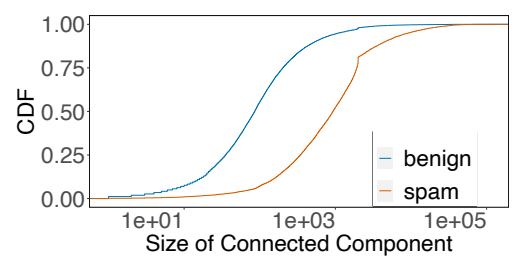

Figure 6: CDF of \# of IPs in a connected component containing a given URL. Spam URLs tend to be in larger connected components. 


\begin{tabular}{l|c|c}
\hline Property & Training Constraints & Robustness \\
\hline \hline Stability & Smaller $c_{\text {stability }}$ & Stronger \\
\hline High Confidence & Smaller $\delta$ & Stronger \\
\hline Redundancy & Smaller $\delta$ & Stronger \\
\hline Small Neighborhood & Smaller $c$ givenfi xed $\epsilon$ & Stronger \\
\hline
\end{tabular}

Table 9: Robustness controlled by hyperparameters.

\begin{tabular}{c|c|c}
\hline Stable Constant $c_{\text {stability }}$ & TPR & FPR \\
\hline \hline 8 & 0.86 & 0.021 \\
\hline 4 & 0.835 & 0.031 \\
\hline 2 & 0.833 & 0.029
\end{tabular}

Table 10: TPR and FPR of Twitter spam account classifiers trained with the stability property.

We measure the distribution of CCSize feature from the Twitter spam URL dataset. The CCSize feature counts the number of IP nodes in the connected component of the posted URL. Since spammers reuse redirectors, their URLs often belong to the same large connected component and result in a larger CCSize feature value, compared to benign URLs, as shown in Figure 6. A larger CCSize value indicates that more resources are being reused, and the initial URL is more suspicious. Therefore, we specify CCSize feature to be monotonically increasing.

\section{CLASSIFICATION FEATURES}

Table 11 lists all the features for detecting cryptojacking, Twitter spam accounts, and Twitter spam URLs.

\section{E HEURISTICS}

The time to solve for counterexamples is the bottleneck in training. Therefore, we implement the following heuristics to improve the training efficiency:

- We exponentially increase the time out for the solver, starting from 30s. If wefi nd at least one counterexample within each CEGIS iteration, we add the constraint(s) to eliminate the counterexample(s) and proceed with CLN training with the constraints. We increase the timeout if the solver could notfi nd any counterexample fast enough in an iteration, and if it also could not verify that the classifier satisfies all the specified properties.

- We implement property boosting as an option to train monotonicity and stability. Property boosting means that we only train the property for the newly added sub-classifier, and keep the previous sub-classifiersfi xed. This works for properties that can be satisfied if every sub-classifiers also satisfy the sub-properties, since our ensemble is a sum ensemble. If every sub-classifier is monotonic for a given feature, the ensemble classifier is also monotonic. Similarly, if every sub-classifier is stable for a given feature by a stable constant $\frac{c}{B}$, the ensemble classifier is stable under stable constant $c$.

- We use feature scheduling to train the high confidence property. Specifically, to run 10 rounds of boosting for either Twitter spam account or Twitter spam URL detection classifiers, wefi rst boost 6 decision trees as the base model without any low-cost features. This makes sure that the base model naturally satisfies the high confidence property. Then, for the remaining 4 rounds, we use all features to boost new trees andfi $\mathrm{x}$ the properties for the entire classifier.

- When training all thefi ve properties (monotonicity, stability, high confidence, redundancy and small neighborhood) for the Twitter spam account detection, we use the following property scheduling to boost 6 rounds. For thefi rst round, we use features that don't involve any property to construct a base classifier, so it naturally satisfies all properties. In the 2 nd and 3rd round, we use all features excluding low-cost ones, so we get high confidence and redundancy for free for these rounds. In the next two rounds, we use all features excluding monotonic ones, so we get monotonicity for free for these rounds. In the last round, we use all features andfi $x$ allfi ve properties.

Property boosting, feature scheduling, and property scheduling reduce the size of the integer linear program, which makes it easier to be solved.

\section{F HYPERPARAMETERS}

Enforcing stronger robustness decreases true positive rate. The hyperparameters control this tradeoff. In particular, Table 9 shows how the strength of robustness changes as different hyperparameters change for all proposed properties except monotonicity (we don't have such a hyperparameter for monotonicity). For example, to demonstrate the tradeoff, we trained three Twitter spam account classifiers with the stability property, where each one has a different stable constant. Table 10 shows that training with a smaller stable constant $c_{\text {stability }}$ gives us a verifiably robust model with stronger robustness but lower true positive rate.

\section{G OBTAINING MORE PROPERTIES}

Table 8 shows that training a classifier with one property sometimes obtains another property. For the Twitter spam account detection classifiers, enforcing one of the high confidence, redundancy, and small neighborhood properties can obtain at least a second property. For example, the Logic Ensemble Redundancy model has obtained stability and high confidence properties. Since we use the same set of low-cost features to define the high confidence and redundancy properties, the redundancy property is strictly stronger than the high confidence property. In other words, if the attacker have to perturb one low-cost feature from at least two different groups to evade the classifier (redundancy), they cannot evade the classifier by perturbing only one low-cost feature (high confidence). For the largest Twitter spam URL detection dataset, the Logic Ensemble Stability model also satisfies the small neighborhood property, and the Logic Ensemble High confidence model also satisfies the stability property.

\section{H LOGIC ENSEMBLE COMBINED MODEL}

Table 8 shows that, for Twitter spam account detection, the Logic Ensemble Combined with allfi ve properties has higher AUC than the Logic Ensemble Monotonicity model trained with only one property. This is because we use property scheduling for Logic Ensemble Combined (Appendix E), such that for each round before the last round, our classifier satisfies some properties for free. We could improve the performance of the Logic Ensemble Monotonicity by similar feature scheduling technique, such as boostingfi rst four 


\begin{tabular}{|c|c|c|c|c|}
\hline Dataset & Feature Name & Description & Monotonic & Low-cost \\
\hline \multirow{7}{*}{ Cryptojacking } & websocket & Use WebSocket APIs for network communication & Increasing & \\
\hline & wasm & Uses WebAssembly to execute code in browsers are near native speed & Increasing & \\
\hline & hash function & Use one of the hash functions on a curated list & Increasing & yes \\
\hline & webworkers & The number of web workers threads for running concurrent tasks & Increasing & \\
\hline & messageloop load & The number of MessageLoop events for thread management & Increasing & \\
\hline & postmessage load & The number of PostMessage events for thread job reporting & Increasing & \\
\hline & parallel functions & Run the same tasks in multiple threads & Increasing & \\
\hline \multirow{15}{*}{$\begin{array}{c}\text { Twitter Spam } \\
\text { Accounts }\end{array}$} & LenScreenName & The number of characters in the account user name & Increasing & yes $(\geq 5$ char $)$ \\
\hline & LenProfileDescription & The number of characters in the profile description & & yes \\
\hline & AgeDays & The age of the account in days & Decreasing & \\
\hline & NumFollowings & The number of other users an account follows & Increasing & \\
\hline & NumFollowers & The number of followers for an account & Decreasing & \\
\hline & Ratio_Following_Followers & The ratio of NumFollowings divided by NumFollowers & & \\
\hline & StdFollowing & Standard deviation of NumFollowings over different days & & \\
\hline & ChangeRateFollowing & The averaged difference for NumFollowings between consecutive days & & \\
\hline & NumTweets & Total number of tweets over seven months & & yes \\
\hline & NumDailyTweets & Average number of daily tweets & & yes \\
\hline & TweetLinkRatio & Ratio of tweets containing links over total number of tweets & Increasing & yes \\
\hline & TweetUniqLinkRatio & Ratio of tweets containing unique links over total number of tweets & Increasing & yes \\
\hline & TweetAtRatio & Ratio of tweets containing ‘@’ over total number of tweets & & yes \\
\hline & TweetUniqAtRatio & Ratio of tweets with unique ‘@’ username over total number of tweets & & yes \\
\hline & PairwiseTweetSimilarity & Normalized avg num of common chars in pairwise tweets for a user & & \\
\hline \multirow{29}{*}{$\begin{array}{l}\text { Twitter Spam } \\
\text { URLs }\end{array}$} & \multicolumn{4}{|l|}{ Shared Resources-driven } \\
\hline & EntryURLid & In degree of the largest redirector in the connected component & Increasing & \\
\hline & AvgURLid & Average in degree of URL nodes in the redirection chain & Increasing & \\
\hline & ChainWeight & Total frequency of edges in the redirection chain & Increasing & \\
\hline & CCsize & Number of nodes in the connected component & Increasing & \\
\hline & CCdensity & Edge density of the connected component & & \\
\hline & MinRCLen & Minimum length of the redirection chains in the connected component & Increasing & \\
\hline & AvgLdURLDom & Avg \# of domains for landing URL IPs in the connected component & Increasing & \\
\hline & AvgURLDom & Average \# of domains for the IPs in the redirection chain & Increasing & \\
\hline & \multicolumn{4}{|l|}{ Heterogeneity-driven } \\
\hline & GeoDist & Total geographical distance $(\mathrm{km})$ traversed by the redirection chain & & \\
\hline & CntContinent & Number of unique continents in the redirection chain & & \\
\hline & CntCountry & Number of unique countries in the redirection chain & & \\
\hline & CntIP & Number of unique IPs in the redirection chain & & \\
\hline & CntDomain & Number of unique domains in the redirection chain & & \\
\hline & CntTLD & Number of unique top-level domains in the redirection chain & & \\
\hline & \multicolumn{4}{|l|}{ Flexibility-driven } \\
\hline & ChainLen & Length of the redirection chain & & \\
\hline & EntryURLDist & Distance from the initial URL to the largest redirector & & \\
\hline & CntInitURL & Number of initial URLs in the connected component & & \\
\hline & CntInitURLDom & Total domain name number in the initial URLs & & \\
\hline & CntLdURL & Number offi nal landing URLs in the redirection chain & & \\
\hline & AvgIPperURL & Average IP number per URL in the connected component & & \\
\hline & AvgIPperLdURL & Average IP number per landing URL in the connected component & & \\
\hline & \multicolumn{4}{|l|}{ Tweet Content } \\
\hline & Mention Count & Number of ‘@’ that mentions other users & & yes \\
\hline & Hashtag Count & Number of hashtags & & yes \\
\hline & Tweet Count & Number of tweets made by the user account for this tweet & & yes \\
\hline & URL Percent & Percentage of posts from the same user that contain a URL & & yes \\
\hline
\end{tabular}

Table 11: Classification features for three datasets. For each feature, we also mark the monotonic direction if we specify the monotonicity property, and whether we specify the feature to be low cost.

rounds of model with non-monotonic featuresfi rst, and then train with all features for later rounds. 